\title{
COFINAL TYPES OF TOPOLOGICAL DIRECTED ORDERS
}

\author{
SŁAWOMIR SOLECKI AND STEVO TODORCEVIC
}

\begin{abstract}
We investigate the structure of the Tukey ordering among directed orders arising naturally in topology and measure theory.
\end{abstract}

\section{INTRODUCTION}

Let $D$ and $E$ be directed partial orders. A function $f: D \rightarrow E$ is called Tukey if the preimage of each bounded subset of $E$ is bounded in $D$. A function $g: E \rightarrow D$ is called convergent if it maps cofinal subsets of $E$ to cofinal subsets of $D$. The existence of a Tukey map from $D$ to $E$ is equivalent to the existence of a convergent map from $E$ to $D$. If this relation holds between $D$ and $E$, we write $D \leq_{T} E$ and say that $D$ is Tukey reducible to $\mathrm{E}$ or $D$ is Tukey below $E$. We write $D \equiv_{T} E$ if $D \leq_{T} E$ and $E \leq_{T} D$. The relation $D \equiv_{T} E$ is equivalent to the existence of a directed partial order into which both $D$ and $E$ embed as cofinal subsets. We say then that $D$ and $E$ realize the same cofinal type [14].

Tukey ordering was introduced by Tukey [14] in an attempt to illuminate the Moore-Smith theory of net convergence. It was then realized that the relation $\leq_{T}$ can be useful in calibrating complexities of various directed sets or, more generally, partially ordered sets, see [5], [10], [11], [14]. As demonstrated by Fremlin in [3], Tukey reducibility is central to understanding the results of Bartoszynski and Raisonnier-Stern saying in effect that the $\sigma$-ideal of meager subsets of $[0,1]$ is more additive than the ideal of Lebesgue measure zero subsets of $[0,1]$. Christensen in [1, Chapter 3] used Tukey reducibility to study the space of compact subsets of metric separable spaces. Through the work above and an earlier paper by Isbell [5], it became apparent that the relation $\leq_{T}$ is particularly interesting when restricted to partially ordered sets occurring in analysis and topology. In the present paper, we continue this line of research.

On closer inspection, it turns out that examples of partial orders studied in this context come from two major classes. The first one is the class of analytic P-ideals of subsets of $\mathbb{N}$ with inclusion, see for example [3], [5], [8], [9], [12], [13]. (By an analytic P-ideal we mean an ideal $I$ of subsets of $\mathbb{N}$ which is analytic as a subspace of $2^{\mathbb{N}}$ and is such that for any $x_{n} \in I, n \in \mathbb{N}$, there is $x \in I$ with $x_{n} \backslash x$ finite

2000 Mathematics Subject Classification. Primary: 03E05, 06A07; Secondary: 03E15, 03E17, $22 \mathrm{~A} 26$.

Key words and phrases. Tukey order, analytic ideals, $\sigma$-ideals of compact sets.

The first author was partially supported by NSF grant DMS-0102254. A large part of this work was done when the first author visited Paris during June and July 2003. The visit was supported by the CNRS-University of Illinois collaboration funds and by University of Paris VI. 
for each $n$.) The second class consists of $\sigma$-ideals of compact sets with inclusion, see for example [1], [3], [4], [7]. (By a $\sigma$-ideal of compact sets we mean a family $I$ of compact subsets of a metric separable space such that if $L \subseteq K \in I$ and $L$ is compact, then $L \in I$, and if $K_{n} \in I, n \in \mathbb{N}$, and $\bigcup_{n} K_{n}$ is compact, then $\bigcup_{n} K_{n} \in I$.) An important element of the first class is the $F_{\sigma} \mathrm{P}$-ideal $\mathcal{I}_{1 / n}$ defined by

$$
\mathcal{I}_{1 / n}=\left\{x \subseteq \mathbb{N}: \sum\left\{\frac{1}{n+1}: n \in x\right\}<\infty\right\} .
$$

It is, in fact, a maximal element with respect to the Tukey ordering among analytic P-ideals. It is known (see [3]) that $\mathcal{I}_{1 / n}$ captures all combinatorial properties related to additivity and cofinality of the ideal of Lebesgue measure zero subsets of $[0,1]$ with inclusion. For this reason, one may call the family of all analytic P-ideals the measure leaf of the Tukey ordering. The second one of these classes, $\sigma$-ideals of compact subsets of separable metric spaces, contains an important partial order NWD of compact nowhere dense subsets of $[0,1]$. It is not Tukey maximal among $\sigma$-ideals of compact subsets of metric separable spaces but it is possible that it is maximal among analytic such $\sigma$-ideals, however, this is still open. Again, it is known (see [3]) that NWD captures all combinatorial properties related to additivity and cofinality of the $\sigma$-ideal of meager subsets of $[0,1]$. One may call the family of all $\sigma$-ideals of compact subsets of metric separable spaces or similar directed orders the category leaf of the Tukey ordering.

We investigate the structure of the Tukey order among partial orders belonging to these two classes. In fact, we define a natural family of partial orders, we call basic orders, containing both these classes, as well as other interesting examples, and establish our results in this greater generality. It is somewhat unexpected that conditions defining this family and allowing our proofs to go through involve only simple interaction between topology and order (each convergent (bounded) sequence has a bounded (convergent) subsequence) and the existence of a continuous maximum operation. We hope that our results provide partial evidence that basic orders form the right framework for investigating the Tukey reduction among directed orders coming from topology and analysis. Here is a summary of the main results.

We show that if the topology on a basic order is analytic, then it is Polish. This generalizes results of Christensen [1] and Kechris, Louveau, and Woodin [7]. As a consequence of it, we prove that analytic P-ideals are the only ideals of subsets of $\mathbb{N}$ which can be made into analytic basic orders by strengthening the topology on them. We prove that functions witnessing Tukey reducibility between basic orders can always be chosen to be definable (Borel or measurable with respect to the smallest $\sigma$-algebra generated by analytic sets). This is new even for analytic P-ideals and $\sigma$-ideals of compact sets and confirms the empirical fact noticed by several people that whenever there was a Tukey reduction or a convergent map between pairs of such directed orders one was able to find an effective such map. Next, we define a general type of property of basic orders which is invariant under Tukey reducibility. Using these general invariants, we are able to establish results 
on Tukey non-reducibility between concrete partial orders. One of these results concerns the relation between the measure and the category leaves. In a sense the first one dominates the second one. The first indication of this is given by NWD $\leq_{T} \mathcal{I}_{1 / n}$ (see [3]) which summarizes several known results of Bartoszynski, Raisonnier-Stern, Pawlikowski, and others mentioned earlier in the introduction. We enrich this theme further by showing that this relation is strict and, in fact, no member of the measure leaf, except those Tukey equivalent with $1, \mathbb{N}$, and $\mathbb{N}^{\mathbb{N}}$, is Tukey below a member of the category leaf. The second non-reducibility result concerns partial orders in the measure leaf, that is, analytic P-ideals. The top element there, $\mathcal{I}_{1 / n}$, is an $F_{\sigma}$ P-ideal. We show that all $F_{\sigma}$ P-ideals are high in the Tukey ordering in this leaf. For example, no such ideal, except those Tukey equivalent to 1 or $\mathbb{N}$, is Tukey below the analytic P-ideal

$$
\mathcal{Z}_{0}=\left\{x \subseteq \mathbb{N}: \lim _{n} \frac{|x \cap\{0,1, \ldots, n\}|}{n+1}=0\right\}
$$

of all subsets of $\mathbb{N}$ of asymptotic density 0 . These results strengthen theorems of Fremlin [3] and Louveau and Veličković [8]. We define and study a functor $I \rightarrow D(I)$ from the measure leaf into the category leaf. We show that $D$ respects the Tukey order, that is, $I \leq_{T} J$ implies $D(I) \leq_{T} D(J)$ for analytic P-ideals $I, J$ with $J$ containing at least one infinite set, we prove that $D(I) \leq_{T} I$ for all analytic P-ideals and establish upper and lower Tukey bounds for $D(I)$. We also show that $D\left(\mathcal{I}_{1 / n}\right)$, the Tukey top element among $D(I)$ for analytic P-ideals $I$, is high in the Tukey order. Finally, sharpening a result of Zafrany [15], we prove that each analytic ideal of subsets of $\mathbb{N}$ has a cofinal subset which is $G_{\delta}$.

\section{StANDARD NOtATION AND DEFInitions}

We recall now definitions of some of the relevant notions. By a Polish space we understand a metric separable complete space. A metric separable space is called analytic if it is the continuous image of a Polish space. If $X$ is a separable metric space, by $\mathcal{K}(X)$ we denote the space of all compact subsets of $X$ (including the empty set) with the Vietoris topology, that is, the topology generated by sets of the form $\{K \in \mathcal{K}(X): K \cap U \neq \emptyset\}$ and $\{K \in \mathcal{K}(X): K \subseteq U\}$ for $U \subseteq X$ open.

The introduction contains definitions and some examples of $\sigma$-ideals of compact sets and of analytic $P$-ideals. $\sigma$-ideals of compact sets will be considered with the Vietoris topology. It was proved in [9] that each analytic P-ideal $I$ is of the form $I=\operatorname{Exh}(\phi)$ where $\phi: \mathcal{P}(\mathbb{N}) \rightarrow[0, \infty]$ is a lower semicontinuous (lsc) submeasure and where

$$
\operatorname{Exh}(\phi)=\left\{x \subseteq \mathbb{N}: \lim _{n} \phi(x \backslash\{0, \ldots, n\})=0\right\} .
$$

For example for $\mathcal{I}_{1 / n}$ and $\mathcal{Z}_{0}$ the lsc submeasures are $\phi_{1}(x)=\sum\{1 /(n+1): n \in x\}$ and $\phi_{2}(x)=\sup _{n}|x \cap\{0,1, \ldots, n\}| /(n+1)$, respectively. If $I=\operatorname{Exh}(\phi)$ for a lsc submeasure $\phi$, then $d(x, y)=\phi(x \triangle y)$ is a metric on $I$. It was proved in [9] that the topology on $I$ induced by this metric is Polish and does not depend on the choice of $\phi$. We will call it the submeasure topology. The topology inherited by $I$ from the inclusion $I \subseteq 2^{\mathbb{N}}$ contains the submeasure topology. It follows easily from (2.1) that 
each analytic P-ideal is in fact $F_{\sigma \delta}$ as a subset of $2^{\mathbb{N}}$. The ideal $\mathcal{I}_{1 / n}$ is a P-ideal which is $F_{\sigma}$ and the ideal of asymptotically density zero sets $\mathcal{Z}_{0}$ is an example of an $F_{\sigma \delta}$ P-ideal which is not $G_{\delta \sigma}$. We consider the improper ideal $\mathcal{P}(\mathbb{N})$ of all subsets of $\mathbb{N}$ to be an analytic P-ideal. The simplest non-trivial analytic P-ideal is the ideal of finite subsets of $\mathbb{N}$ denoted by Fin. We call an ideal $I$ a trivial modification of Fin if, for some $a \subseteq \mathbb{N}, I=\{x \subseteq \mathbb{N}: x \cap a$ is finite $\}$. Our convention is that each ideal of subsets of $\mathbb{N}$ contains all singletons of natural numbers.

If $D$ is a partial order, we frequently denote the partial order relation on $D$ by $\leq_{D}$. A subset $A \subseteq D$ is called cofinal if for any $x \in D$ there is $y \in A$ with $x \leq_{D} y$. For partial orders $D$ and $E$, we call a function $f: D \rightarrow E$ monotone if, for all $x_{1}, x_{2} \in D, x_{1} \leq_{D} x_{2}$ implies $f\left(x_{1}\right) \leq_{E} f\left(x_{2}\right)$.

For two partial orders $D$ and $E$, we write $D<_{T} E$ if $D \leq_{T} E$ and $E \not_{T} D$.

\section{BASIC DEFINITIONS AND LEMMAS}

We present here a general definition of a family of partial orders. It will turn out that this family includes all relevant to us partial orders. Here and in the sequel when we say that a set is bounded, we mean bounded from above; when we say an element is a bound for a set, we mean it is an upper bound.

Definition. Let $D$ be a separable metric space and let $\leq$ be a partial order on $D$. We say that $(D, \leq)$ is basic if

(1) each pair of elements of $D$ has the least upper bound with respect to $\leq$ and the binary operation of least upper bound from $D \times D$ to $D$ is continuous;

(2) each bounded sequence has a converging subsequence;

(3) each converging sequence has a bounded subsequence.

Note that (1) implies that each finite subset $F$ of a basic order $D$ has the least upper bound which we will denote by $\max F$.

The following classes are the main groups of examples of basic orders.

Examples. $\quad$ 1. Analytic P-ideals. Let $I$ be an analytic P-ideal. Let $\phi$ be a lsc submeasure with $I=\operatorname{Exh}(\phi)$. Then $(I, \subseteq)$ with the submeasure topology is a basic order. Condition (1) is easily checked from the definition of the topology on $I$. Condition (2) also follows since, as is easily verified, for any $x \in I$, on the set $\{y: y \subseteq x\}$ the submeasure topology and the topology inherited from $2^{\mathbb{N}}$ coincide and the latter topology is compact. To see (3), let a sequence $\left(x_{n}\right)$ of elements of $I$ converge to $x \in I$. Then $\lim _{n} \phi\left(x_{n} \triangle x\right)=0$. Thus, we can pick a subsequence $\left(x_{n_{k}}\right)$ of $\left(x_{n}\right)$ so that $\sum_{k} \phi\left(x_{n_{k}} \triangle x\right)<\infty$. But then one easily checks that $\bigcup_{k} x_{n_{k}} \in \operatorname{Exh}(\phi)=I$ and this set is a bound of the subsequence $\left(x_{n_{k}}\right)$.

2. $\sigma$-ideals of compact sets. Let $I$ be a $\sigma$-ideal of compact subsets of a separable metric space with the Vietoris topology and with inclusion as the partial order. Then $(I, \subseteq)$ is a basic order. Condition (1) is checked from the definition of the Vietoris topology. Condition (2) follows from the wellknown fact, that for a compact $L, \mathcal{K}(L)$ is compact. If $\left(K_{n}\right)$ is a sequence 
of elements of $I$ converging to $K \in I$, then $\bigcup_{n} K_{n} \cup K$ is compact and, therefore, belongs to $I$ and is a bound for the whole sequence $\left(K_{n}\right)$. Thus, (3) follows.

3. Relative $\sigma$-ideals of compact sets. The class of examples from point 2 can be widened to include some basic orders we will come across in this paper (Section 7). Let $\mathcal{K}$ be a closed family of compact subsets of a separable metric space. Let $I \subseteq \mathcal{K}$ be such that if $L \subseteq K \in I, L \in \mathcal{K}$, then $L \in I$ and if $K_{n} \in I, n \in \mathbb{N}$, and $\bigcup_{n} K_{n} \in \mathcal{K}$, then $\bigcup_{n} K_{n} \in I$. In this situation, by the same arguments as in point $2,(I, \subseteq)$ is a basic order. (Closedness of $\mathcal{K}$ is used in checking (2).) We call families $I$ of compact sets as above relative $\sigma$-ideals of compact sets.

Note that we could have defined the objects of our study to be topological Abelian semigroups with metric separable topology and with each element idempotent. (The semigroup operation + would correspond to taking the least upper bound.) Then we could define the partial order in the usual way by setting $x \leq y \Leftrightarrow x+y=y$ and assume conditions (2) and (3) about so defined $\leq$. One of the reasons we prefer to use a partial order as our primitive operation is the fact that the role the least upper bound, with property (1), plays in our proofs is auxiliary - it is used to establish, in the two lemmas below, stronger versions of conditions (2) and (3) and only these strengthened conditions are used directly in all our proofs except one (Theorem 5.1).

Lemma 3.1. Let $D$ be a basic order. Then, for each $x \in D,\left\{y \in D: y \leq_{D} x\right\}$ is compact.

Proof. It is obvious from (1) that $\leq_{D}$ is closed. Thus, for each $x \in D,\{y \in D$ : $\left.y \leq_{D} x\right\}$ is closed and the conclusion follows immediately from (2).

Lemma 3.2. Let $D$ be a basic order. Let $x \in D$, and let $x_{k}^{n} \in D, k, n \in \mathbb{N}$, be such that, for each $n, \lim _{k} x_{k}^{n}$ exists and is bounded by $x$. Then any open set $U \ni x$ contains an element $z$ such that for each $n$ the set $\left\{k: x_{k}^{n} \leq z\right\}$ is infinite.

Proof. Claim. Given a sequence $\left(x_{n}\right)$ of elements of $D$ converging to $x \in D$ and an open set $U \ni x$, there exists $z \in U$ which bounds a subsequence of $\left(x_{n}\right)$.

Proof of claim. By condition (3), there exists a $y \in D$ which bounds a subsequence of $\left(x_{n}\right)$. To make our notation simpler, we assume that $y$ bounds all the elements of $\left(x_{n}\right)$. Let $V$ be an open set containing $x$ and whose closure is included in $U$. Now continuity of the maximum operation, i.e., (1), allows us to pick a subsequence $\left(x_{n_{k}}\right)$ of $\left(x_{n}\right)$ so that, for each $m, x^{m}=\max \left\{x_{n_{k}}: k \leq m\right\} \in V$. Obviously, $x^{m} \leq y$ for each $m$ and, therefore, by $(2),\left(x^{m}\right)$ contains a subsequence which converges to a $z \in \bar{V} \subseteq U$. Note that continuity of the maximum operation implies that $\leq$ is closed from which it follows that $z$ bounds all the elements of $\left(x_{n_{k}}\right)$, which finishes the proof of the claim.

Define now

$$
y_{k}^{m}=\max \left(\{x\} \cup\left\{x_{k}^{n}: n \leq m\right\}\right) .
$$


By the assumption on the sequences $\left(x_{k}^{n}\right)_{k}, n \in \mathbb{N}$, and continuity of the maximum operation, we get that, for each $m, \lim _{k} y_{k}^{m}=x$. The claim implies now that there exist $y_{m} \in D, m \in \mathbb{N}$, such that $y_{m} \rightarrow x$ and for each $m$

$$
\left\{k: y_{k}^{m} \leq y_{m}\right\} \text { is infinite. }
$$

Applying the claim once again, to the sequence $\left(y_{m}\right)$ converging to $x$ this time, we get a $z \in U$ such that $\left\{m: y_{m} \leq z\right\}$ is infinite. This, in combination with (3.1), makes it possible to chose strictly increasing sequences $\left(m_{l}\right)$ and $\left(k_{l}\right)$ so that, for each $l$,

$$
y_{k_{l}}^{m_{l}} \leq z
$$

This inequality in conjunction with the definition of the $y_{k}^{m}$ 's immediately imply that $z$ fulfills the conclusion of the lemma.

As we already mentioned, in most of our results we only need that the involved partial orders fulfill conclusions of Lemma 3.1 or Lemma 3.2. In fact, frequently one can get by with even slightly weaker assumptions. However, we will usually state simply that the partial orders are basic and leave it to the reader to find out precisely what is used. We make an exception to this rule when it suffices to assume the conclusion of Lemma 3.1, that is, that the set of predecessors of each element is compact. We do this since there are many examples of partial orders of some interest fulfilling this condition without necessarily satisfying the conclusion of Lemma 3.2, for instance, all ideals of subsets of $\mathbb{N}$ and all ideals of compact subsets of metric separable spaces.

\section{Analytic Basic ORDERS}

A basic order $(D, \leq)$ is called analytic if the metric space $D$ is analytic (i.e., $D$ is a continuous image of a Polish space). Most of the classically studied P-ideals and $\sigma$-ideals of compact sets are analytic. First, we have a theorem on topologies on analytic basic orders.

Theorem 4.1. If $D$ is an analytic basic order, then the topology on $D$ is Polish.

Proof. Let $D$ be a an analytic basic order. Let $X$ be a metric compactification of $D$. Fix a continuous surjection $f: \mathbb{N}^{\mathbb{N}} \rightarrow D$ and a countable topological basis $\mathcal{U}$ of $X$ with $X \in \mathcal{U}$. Let $\mathbb{N}^{<\mathbb{N}}$ be the set of all finite sequences of natural numbers. For $s \in \mathbb{N}^{\mathbb{N}}$ let $|s|$ stand for the length of $s$. For $s, t \in \mathbb{N}^{<\mathbb{N}}$, we write $s \leq_{0} t$ if $|s|=|t|$ and $s(i) \leq t(i)$ for all $i<|s|$. We call a pair $(W, s) \operatorname{good}$ if $W \in \mathcal{U}, s \in \mathbb{N}^{<\mathbb{N}}$ and

$$
\forall d \in D\left(d \in W \Rightarrow \exists x \in \mathbb{N}^{\mathbb{N}} d \leq_{D} f(x) \text { and } x|| s \mid \leq_{0} s\right) .
$$

Note that there are only countably many good pairs.

For $s \in \mathbb{N}^{<\mathbb{N}}$ and $m \in \mathbb{N}$, let $\hat{s} m$ be the sequence $s$ followed by $m$. We claim that for $d \in X$ the following equivalence holds:

$$
d \in D \Leftrightarrow \forall(W, s) \operatorname{good}(d \notin W \text { or } \exists m \in \mathbb{N} \exists U(U, \hat{s} m) \text { good, } U \subseteq W, d \in U) \text {. }
$$

Note that this equivalence defines $D$ as a $G_{\delta}$ subset of $X$ which will prove the theorem. It remains, therefore, to establish the above equivalence. 
We prove first the implication from right to left. Fix $d \in X$ fulfilling the condition on the right. After noting that $(X, \emptyset)$ is good, this condition implies that we can find $y \in \mathbb{N}^{\mathbb{N}}$ and a descending sequence $\left(U_{n}\right)$ of elements of $\mathcal{U}$ with $X=U_{0}$ such that, for each $n, d \in U_{n}$ and $\left(U_{n}, y \mid n\right)$ is good. Now, the definition of goodness and density of $D$ in $X$ give sequences $y_{n} \in \mathbb{N}^{\mathbb{N}}$ and $d_{n} \in D, n \in \mathbb{N}$, such that $y_{n}\left|n \leq_{0} y\right| n, d_{n} \leq_{D} f\left(y_{n}\right)$, and $d_{n} \rightarrow d$. The first one of these conditions implies that, after going to a subsequence, we can assume that $\left(y_{n}\right)$ converges which, by continuity of $f$, gives as a consequence convergence of the sequence $\left(f\left(y_{n}\right)\right)$ to an element of $D$. This, along with condition (3) from the definition of basic orders, produces $d_{\infty} \in D$ with $f\left(y_{n}\right) \leq_{D} d_{\infty}$, for infinitely many $n$, which obviously implies that $d_{n} \leq_{D} d_{\infty}$ for infinitely many $n$. Combining this last consequence with (2) from the definition of basic orders, we obtain a subsequence of $\left(d_{n}\right)$ converging to an element of $D$. But $\left(d_{n}\right)$ converges to $d$, whence $d \in D$.

To prove the implication from left to right fix a $d \in D$. We will show the condition on the right. In order to do this, fix $(W, s)$ good with $d \in W$. Now suppose, towards contradiction, that for no $U \in \mathcal{U}$ and no $m \in \mathbb{N}$ we have $d \in U \subseteq W$ and $\left(U, s^{\wedge} m\right)$ good. By considering elements $U$ of $\mathcal{U}$ containing $d$ and of smaller and smaller diameter, this allows us to construct a sequence $d_{n} \in D, n \in \mathbb{N}$, converging to $d$ and such that

$$
\neg\left(\exists y \in \mathbb{N}^{\mathbb{N}} \exists n \in \mathbb{N}\left(y \mid(|s|+1) \leq_{0} \hat{s} n \text { and } d_{n} \leq_{D} f(y)\right)\right) .
$$

Since $\left(d_{n}\right)$ converges to $d$, by Lemma 3.2 , we can find $d^{\prime} \in W \cap D$ such that

$$
\left\{n \in \mathbb{N}: d_{n} \leq_{D} d^{\prime}\right\} \text { is infinite. }
$$

Since $(W, s)$ is good, there exists a $y_{0} \in \mathbb{N}^{\mathbb{N}}$ such that $d^{\prime} \leq_{D} f\left(y_{0}\right)$ and $y_{0}|| s \mid \leq_{0} s$. Find now $n_{0}$ in the infinite set in (4.2) with $y_{0}(|s|) \leq n_{0}$. Then, $d_{n_{0}} \leq_{D} d^{\prime} \leq_{D} f\left(y_{0}\right)$ and $y_{0} \mid(|s|+1) \leq s^{\wedge} n_{0}$ which directly contradicts (4.1).

We note that for the conclusion of the theorem above to hold, it is enough to suppose only that any bounded sequence in $D$ has a convergent subsequence (i.e., condition (2) of the definition of basic orders) and that for any sequence $\left(x_{n}\right)$ of elements of $D$ converging to $x \in D$ and any open set $U \ni x$ there is a subsequence of $\left(x_{n}\right)$ bounded by an element of $U$.

In connection with Theorem 4.1, one should mention that analyticity among $\sigma$-ideals of compact sets was first studied by Christensen. In [1, Theorem 3.3], he proved in effect that the following conditions are equivalent for a separable metric space $X: \mathcal{K}(X) \leq_{T} \mathbb{N}^{\mathbb{N}}, \mathcal{K}(X)$ is analytic, and $\mathcal{K}(X)$ is Polish. The equivalence of the last two conditions in this theorem was generalized by Kechris, Louveau, and Woodin who proved in [7] that each analytic $\sigma$-ideal of compact subsets is Polish. On the side of analytic P-ideals, a related fact can be read off the results in [9]. The submeasure topology defined in this paper on an analytic P-ideal which makes the ideal into a basic order is always Polish. Thus, Theorem 4.1 above generalizes these results and puts them in a wider context. The basic idea of the proof of this theorem is still reminiscent of that of Christensen's. 
As an application of the above general Theorem 4.1, we show that analytic Pideals are the only ideals of subsets of $\mathbb{N}$ which can be made into basic orders by strengthening the topology on them inherited from $2^{\mathbb{N}}$.

Corollary 4.2. Let $I$ be an ideal of subsets of $\mathbb{N}$. Let $\tau$ be a topology on I such that

(i) $\tau$ contains the topology $I$ inherits from the inclusion $I \subseteq 2^{\mathbb{N}}$;

(ii) $(I, \subseteq)$ with $\tau$ is an analytic basic order.

Then $I$ is an analytic P-ideal and $\tau$ is the submeasure topology.

Proof. Let $\tau$ be a topology on $I$ with (i) and (ii). We show that the symmetric difference on $I$ is continuous with respect to $\tau$. In order to accomplish this, it will be enough to prove that for any two sequences $\left(x_{n}\right),\left(y_{n}\right)$ of elements of $I$ converging to $x$ and $y$, respectively, there exists a subsequence of $\left(x_{n} \triangle y_{n}\right)$ converging to $(x \triangle y)$. Since $(I, \subseteq)$ with $\tau$ is basic, by applying condition (2) twice, we can find an increasing sequence $\left(n_{k}\right)$ and $x^{\prime}, y^{\prime} \in I$ such that $x^{\prime}$ bounds all elements of $\left(x_{n_{k}}\right)$ and $y^{\prime}$ bounds all elements of $\left(y_{n_{k}}\right)$. Note that $\mathcal{P}\left(x^{\prime} \cup y^{\prime}\right)$ is included in I. Again, since $\tau$ makes $(I, \subseteq)$ basic, by Lemma 3.1, $\mathcal{P}\left(x^{\prime} \cup y^{\prime}\right)$ is compact with the restriction of $\tau$ to it. Thus, there is a further subsequence of $\left(x_{n_{k}} \triangle y_{n_{k}}\right)$ which is convergent. We denote this subsequence again by $\left(x_{n_{k}} \triangle y_{n_{k}}\right)$. Since $x_{n_{k}} \triangle y_{n_{k}}$ converges to $x \triangle y$ in the topology on $2^{\mathbb{N}}$ and $\tau$ contains this topology, we see that it follows that $x_{n_{k}} \triangle y_{n_{k}} \rightarrow x \triangle y$ with respect to $\tau$ as $k \rightarrow \infty$, and we have proved that $\triangle$ is a continuous operation with respect to $\tau$.

Now, by Theorem 4.1, $I$ with $\tau$ is Polish. It was proved in [9] that if $\tau$ is a Polish topology on an ideal $I$ fulfilling (i) and making $\triangle$ continuous (that is, $I$ with $\tau$ and $\triangle$ is a Polishable group), then $I$ is an analytic P-ideal and the topology $\tau$ is the submeasure topology.

The following proposition concerns the bottom part of the Tukey ordering among analytic basic orders. It should be compared with [4, Theorem 15] parts of which it generalizes.

Proposition 4.3. Let $D$ be a non-empty basic order.

(i) $D$ is compact if, and only if, $D \equiv_{T} 1$.

(ii) If $D$ is analytic and not locally compact, then $\mathbb{N}^{\mathbb{N}} \leq_{T} D$.

Lemma 4.4. Let $D$ be basic. Assume there exists a compact set $L \subseteq D$ such that each finite subset of $A$ has an upper bound in L. Then $A$ is bounded.

Proof. Let $\left\{x_{n}: n \in \mathbb{N}\right\}$ be a dense subset of $A$. Let $y_{m} \in L$ be an upper bound of $\left\{x_{n}: n \leq m\right\}$. Since $L$ is compact, $\left(y_{m}\right)$ has a converging subsequence which, by condition (3) of the definition of basic orders, has a further subsequence which is bounded by, say, $z \in D$. Then clearly $z$ is an upper bound of the whole sequence $\left(x_{n}\right)$. Since the set $\{x \in D: x \leq z\}$ is compact by Lemma 3.1, $z$ is a bound for $A=\overline{\left\{x_{n}: n \in \mathbb{N}\right\}}$.

Proof of Proposition 4.3. The implication $\Leftarrow$ in (i) is a consequence of Lemma 3.1. The other implication follows immediately from Lemma 4.4 by taking $A=L=D$. 
Now, we prove (ii). Let $X$ be a metric compactification of $D$ and let $d$ be a metric on $X$. Since $D$ is not locally compact, $X \backslash D$ is not closed. Pick $y_{n} \in X \backslash D$ with $y_{n} \rightarrow z \in D$ as $n \rightarrow \infty$. Pick sequences $\left(x_{k}^{n}\right)_{k}, n \in \mathbb{N}$, with $x_{k}^{n} \in D, x_{k}^{n} \rightarrow y_{n}$ as $k \rightarrow \infty$, and $d\left(x_{k}^{n}, y_{n}\right)<1 /(n+1)$ for each $k \in \mathbb{N}$. Define $f: D \rightarrow \mathbb{N}^{\mathbb{N}}$ by letting

$$
f(x)(n)=\max \left\{k+1: x_{k}^{i} \leq_{D} x \text { and } i \leq n\right\}
$$

with the convention that $\max \emptyset=0$. Note that for each $x \in D$, for each $n$, $\left\{k \in \mathbb{N}: x_{k}^{n} \leq_{D} x\right\}$ is finite since otherwise, by compactness of $\left\{y \in D: y \leq_{D} x\right\}$, $y_{n}$ would be an element of $D$. Thus, $f$ is well-defined. The function $f$ is clearly monotone.

If $D$ is analytic, it follows from it that the range $f(D)$ is a directed analytic subset of $\mathbb{N}^{\mathbb{N}}$. Further, for each $x \in D, f(x)$ is an increasing element of $\mathbb{N}^{\mathbb{N}}$. Thus, to see that $f(D)$ is Tukey equivalent to $\mathbb{N}^{\mathbb{N}}$, it will suffice to show that for any $\left(k_{n}\right)$ in $\mathbb{N}^{\mathbb{N}}$ there is an $x \in D$ with $f(x)(n)>k_{n}$ for infinitely many $n$. Fix $\left(k_{n}\right)$. The sequence $\left(x_{k_{n}}^{n}\right)$ converges in $D$ to $z$. Therefore, by condition (3), there is an $x \in D$ which bounds a subsequence of $\left(x_{k_{n}}^{n}\right)$. The definition of $f$ immediately implies that, for infinitely many $n, f(x)(n)>k_{n}$.

\section{Effective cofinal, COnvergent, and Tukey maps}

Let $D$ and $E$ be directed partial orders. A function $f: D \rightarrow E$ is called cofinal if it is monotone and has cofinal range. Note that a cofinal function is convergent.

A curious "empirical" fact about maps witnessing Tukey reducibility between pairs of analytic P-ideals or $\sigma$-ideals of compact sets was that if they existed at all, there always was one such map which was simply definable. We show here that there is a theorem behind this fact: Tukey reducibility between basic orders is always witnessed by Borel cofinal maps. (For an analogous result concering Tukey maps, see Theorem 5.3.) Thus, two points are made by this result. First, that a map witnessing Tukey reducibility, which in general is arbitrary, can be found definable if the partial orders involved are basic. Second, that a map witnessing Tukey reducibility can be found to be cofinal. The existence of a cofinal map between two (not necessarily basic) partial orders implies the existence of a convergent and the existence of a Tukey map but, in general, these implications cannot be reversed.

Theorem 5.1. Let $D$ and $E$ be basic orders. Assume $D \leq_{T} E$. Then there exists a cofinal map from $E$ to $D$ which is Borel.

We start with a lemma. Recall that a function $f: X \rightarrow \mathcal{K}(Y), X, Y$ topological spaces, is called upper semicontinuous if $f^{-1}(\{K \in \mathcal{K}(Y): K \subseteq U\})$ is open in $X$ for any open $U \subseteq Y$.

Lemma 5.2. Let $D$ be a metric separable space with a partial order in which the set of predecessors of each element is compact. Let $E$ be a basic order. If $D \leq_{T} E$, then there exists an upper semicontinuous function $h: E \rightarrow \mathcal{K}(D)$ such that

(i) $h(z)$ is bounded in $D$ for any $z \in E$;

(ii) if $z_{1}, z_{2} \in E$ and $z_{1} \leq_{E} z_{2}$, then $h\left(z_{1}\right) \subseteq h\left(z_{2}\right)$;

(iii) $\bigcup h[E]=D$. 
Proof. Let $f: D \rightarrow E$ be a Tukey map. Define

$$
R=\overline{\left\{(x, y) \in D \times E: f(x) \leq_{E} y\right\}} .
$$

Claim. For any $z \in E, \bigcup_{y \leq_{E} z} R^{-1}(y)$ is bounded.

Proof of claim. Pick a sequence $\left\{x_{n}: n \in \mathbb{N}\right\} \subseteq \bigcup_{y \leq_{E} z} R^{-1}(y)$ dense in $\bigcup_{y \leq_{E} z} R^{-1}(y)$. The definition of $R$ allows us to pick, for each $n$, a sequence $\left(\left(x_{k}^{n}, y_{k}^{n}\right)\right)_{k}$ with

$$
\lim _{k}\left(x_{k}^{n}, y_{k}^{n}\right)=\left(x_{n}, y\right)
$$

where $y \in E$ is such that $y \leq_{E} z$, and $\left(x_{k}^{n}, y_{k}^{n}\right)$ are such that $f\left(x_{k}^{n}\right) \leq_{E} y_{k}^{n}$ for each $k$. By Lemma 3.2 applied to the double sequence $\left(y_{k}^{n}\right)_{(n, k)}$, we can find, for each $n$, an increasing sequence $\left(k_{l}^{n}\right)_{l}$ to guarantee that the set $\left\{y_{k_{l}^{n}}^{n}: n, l \in \mathbb{N}\right\}$ is bounded in $E$. Since $f$ is Tukey and $f\left(x_{k}^{n}\right) \leq y_{k}^{n}$ for all $k, n$, we see that $\left\{x_{k_{l}^{n}}^{n}: n, l \in \mathbb{N}\right\}$ is bounded in $D$ by some $x$. Since $\left\{x^{\prime} \in D: x^{\prime} \leq_{D} x\right\}$ is compact, it follows that, for each $n, x_{n} \leq_{D} x$. Using again compactness of $\left\{x^{\prime} \in D: x^{\prime} \leq_{D} x\right\}$ and the way $\left\{x_{n}: n \in \mathbb{N}\right\}$ was chosen, we get that $\bigcup_{y \leq z} R^{-1}(y)$ is bounded by $x$, and the claim is proved.

Now note that for any $z \in E$ the set $\left\{z^{\prime} \in E: z^{\prime} \leq_{E} z\right\}$ is compact. Since $R$ is closed, it follows that, for each $z \in E, \bigcup_{y \leq_{E} z} R^{-1}(y)$ is closed and, therefore, being bounded by the claim, it is compact. It follows that

$$
h(z)=\bigcup_{y \leq_{E} z} R^{-1}(y)
$$

is a function from $E$ to the subspace of $\mathcal{K}(D)$ consisting of all compact bounded subsets of $D$. Moreover, we clearly have for all $z_{1}, z_{2} \in E$

$$
z_{1} \leq_{E} z_{2} \Rightarrow h\left(z_{1}\right) \subseteq h\left(z_{2}\right) .
$$

Note also that $x \in h(f(x))$. Thus, conditions (i)-(iii) hold.

It remains to check that $h$ is upper semicontinuous. Fix $F \subseteq D$ closed. We need to check that the set $\{z \in E: h(z) \cap F \neq \emptyset\}$ is closed in $E$. Let $\left(z_{n}\right)$ be a sequence of elements of this set converging to $z$. We then can find $y_{n} \leq_{E} z_{n}$ and $x_{n} \in F$ with $\left(x_{n}, y_{n}\right) \in R$. Since $\leq_{E}$ and $R$ are closed, it will suffice to find an increasing sequence $\left(n_{k}\right)$ such that $\left(y_{n_{k}}\right)$ converges to a $y \in E$ and $\left(x_{n_{k}}\right)$ converges to an $x \in D$. (Then $x \in F, y \leq_{E} z$, and $(x, y) \in R$, so $x \in F \cap h(z)$.) Since $\left(z_{n}\right)$ is convergent, there is a $z^{\prime} \in E$ of which we can assume, by going to a subsequence of $\left(z_{n}\right)$, that $z_{n} \leq_{E} z^{\prime}$ for all $n$ and so, by (ii), $h\left(z_{n}\right) \subseteq h\left(z^{\prime}\right)$. But then the entries of the sequence $\left(y_{n}\right)$ are contained in the compact set $\left\{y \in E: y \leq_{E} z^{\prime}\right\}$ and the entries of $\left(x_{n}\right)$ are contained in the compact set $h\left(z^{\prime}\right)$. Thus, the sequence $\left(n_{k}\right)$ can be found.

Proof of Theorem 5.1. Let $\mathcal{K}_{b}(D)$ stand for the subspace of $\mathcal{K}(D)$ consisting of all non-empty compact bounded subsets of $D$. Let $h$ be a function as in Lemma 5.2. By modifying $h$ to the function $E \ni z \rightarrow h(z) \cup\left\{x_{0}\right\}$, for some fixed $x_{0} \in D$, we can assume that the values of $h$ are non-empty. (We use directedness of $D$ here to make sure that the values of the modified function are still bounded.) Thus, we have a Borel map $h: E \rightarrow \mathcal{K}_{b}(D)$ which is monotone (when compact bounded subsets of 
$D$ are considered with the partial order of inclusion) and such that each element of $D$ is included in a compact set in the range of $h$.

By the Kuratowski-Ryll Nardzewski theorem (see [6]), there exist Borel functions $f_{n}: \mathcal{K}(D) \backslash\{\emptyset\} \rightarrow D, n \in \mathbb{N}$, such that for each $K \in \mathcal{K}(D) \backslash\{\emptyset\}, f_{n}(K) \in K$, for each $n \in \mathbb{N}$, and $\left\{f_{n}(K): n \in \mathbb{N}\right\}$ is dense in $K$. For $K \in \mathcal{K}_{b}(D)$ define

$$
F_{n}(K)=\max \left\{f_{i}(K): i \leq n\right\} .
$$

Fix now $K \in \mathcal{K}_{b}(D)$. Since each subsequence of the sequence $\left(\left\{f_{i}(K): i \leq n\right\}\right)_{n}$ has union which is dense in $K$ and since $\leq_{D}$ is closed, it follows that each limit point of the sequence $\left(F_{n}(K)\right)$ is a bound of $K$ and so a bound of the whole sequence $\left(F_{n}(K)\right)$. On the other hand, the sequence $\left(F_{n}(K)\right)$ is bounded and, hence, included in a compact set and, therefore, each of its subsequences has a limit point. It follows that $\left(F_{n}(K)\right)$ converges in $D$ to a bound of $K$.

Based on the argument in the paragraph above, we can define $f: E \rightarrow D$ by letting

$$
f(z)=\lim _{n} F_{n}(h(z)) .
$$

Since $h$ is Borel, so is $f$. Since each bound of $K$ is above each $F_{n}(h(z))$, for each $n \in \mathbb{N}$, we see that $f(z)$ is the least upper bound of $h(z)$. Thus, it follows from Lemma 5.2(ii) that $f$ is monotone, and from Lemma 5.2(iii) that it is cofinal.

The following theorem complements Theorem 5.1. The partial order $D$ in it and in the corollary following it come from a class of orders wider than basic. For example, $D$ can be an arbitrary ideal of subsets of $\mathbb{N}$ or of compact subsets of a metric separable space. On the other hand, $E$ has to be assumed analytic.

Theorem 5.3. Let $D$ be a metric separable space with a partial order in which the set of predecessors of each element is compact. Let $E$ be an analytic basic order. If $D \leq_{T} E$, then

(i) there exists a Tukey map from $D$ to $E$ measurable with respect to the $\sigma$ algebra generated by analytic sets and

(ii) there exists a convergent map from $E$ to $D$ measurable with respect to the $\sigma$-algebra generated by analytic sets.

Proof. In this proof, when we say that a function is $\Sigma$-measurable, we mean that it is measurable with respect to the $\sigma$-algebra generated by analytic sets.

Let $h$ be a Borel function from $E$ to compact bounded subsets of $D$ as in Lemma 5.2 .

(i) Since $h$ is Borel, the set

$$
P=\{(x, y) \in D \times E: x \in h(y)\}
$$

is Borel as well. Now, the properties of $h$ imply that for each $x \in D$ there is $y \in E$ with $(x, y) \in P$. The Jankov-von Neumann theorem (see [6]) gives a $\Sigma$-measurable uniformization of $P$, that is, we get a $\Sigma$-measurable function $g: D \rightarrow E$ such that

$$
x \in h(g(x)) \text { for all } x \in D .
$$


We claim that $g$ is a Tukey map. Indeed, fix $y \in E$. We only need to see that the set

$$
\left\{x \in D: g(x) \leq_{E} y\right\}
$$

is bounded in $D$. But $g(x) \leq_{E} y$ implies by the properties of $h$ that $h(g(x)) \subseteq h(y)$. Thus, by (5.1), the set above is included in $h(y)$ which is bounded.

(ii) Consider the set

$$
Q=\left\{(y, x) \in E \times D: \forall z \in h(y) z \leq_{D} x\right\} .
$$

By the Kuratowski-Ryll Nardzewski theorem (see [6]) there is a sequence of Borel functions $f_{n}: \mathcal{K}(D) \backslash\{\emptyset\} \rightarrow D, n \in \mathbb{N}$, such that for each $K \in \mathcal{K}(D) \backslash\{\emptyset\}$, $f_{n}(K) \in K$ and $\left\{f_{n}(K): n \in \mathbb{N}\right\}$ is dense in $K$. Since for each $x \in D$, the set $\left\{z \in D: z \leq_{D} x\right\}$ is compact, it follows that

$$
Q=\left\{(y, x) \in E \times D: \forall n \in N f_{n}(h(y)) \leq_{D} x\right\} .
$$

Thus, $Q$ is Borel. Note also that since, for each $y \in E, h(y)$ is bounded, for any $y \in E$ there is an $x \in D$ with $(y, x) \in Q$. By the von Neumann-Jankov theorem there exists a $\Sigma$-measurable uniformization $g$ of $Q$, that is, a function $g: E \rightarrow D$ such that

$$
\forall y \in E \forall z \in h(y) z \leq_{D} g(y) .
$$

We claim that $g$ is a convergent function. Let $A \subseteq E$ be cofinal. We need to see that $g(A)$ is cofinal in $D$. Pick $x \in D$. By the properties of $h$, there is a $y_{1} \in E$ with $x \in h\left(y_{1}\right)$. Let $y_{2} \in A$ be such that $y_{1} \leq_{E} y_{2}$. Then, since $h\left(y_{1}\right) \subseteq h\left(y_{2}\right)$, we have $x \in h\left(y_{2}\right)$. Now, (5.2) implies $x \leq_{D} h\left(y_{2}\right)$ and we are done.

We record now a corollary to Lemma 5.2 showing that the property of being analytic is an invariant for Tukey reducibility. It can be viewed as sharpening of the result of Christensen mentioned after the proof of Theorem 4.1 that $\mathcal{K}(X) \leq_{T} \mathbb{N}^{\mathbb{N}}$ implies that $\mathcal{K}(X)$ is analytic.

Corollary 5.4. Let $D$ be a metric separable space with a partial order in which the set of predecessors of each element is compact. Let $E$ be a basic order. If $D \leq_{T} E$ and $E$ is analytic, then $D$ is analytic as well.

Proof. Let $h: E \rightarrow \mathcal{K}(D)$ be a function as in Lemma 5.2, and let $X$ be a metric compactification of $D$. By upper semicontinuity of $h$, the set $\{(x, y) \in X \times E: x \in$ $h(y)\}$ is closed in $X \times E$ and, therefore, analytic since $E$ is analytic. Now $D$ is the projection of this set along $E$ whereby it is also analytic.

\section{INVARIANTS OF TUKEY REDUCIBILITY}

We define in this section a general type of property of basic orders which is preserved downwards with respect to Tukey order. We deduce from it concrete non-Tukey reducibility results concerning analytic P-ideals and $\sigma$-ideals of compact sets. 
Let $D$ be a metric separable space and let $H$ be a family of subsets of $\mathbb{N}$. We say that $x \in D$ is an $H$-accumulation point of a sequence $\left(x_{n}\right)$ if for each $U$ open containing $x,\left\{n \in \mathbb{N}: x_{n} \in U\right\} \in H$.

Let $D$ be a metric separable space with a partial order. Let $H$ and $I$ be families of subsets of $\mathbb{N}$ with $H$ closed under taking supersets and subtracting finite sets. We say that $D$ is $(H, I)$-calibrated if for each sequence $\left(x_{n}\right)$ of elements of $D$ which has an $H$-accumulation point there is $A \in H$ such that for any $B \in I,\left\{x_{n}: n \in A \cap B\right\}$ is bounded.

We will apply the general Theorem 6.1 to obtain results on Tukey non-reducibility among analytic P-ideals and $\sigma$-ideals of compact sets. The following definitions will be relevant. By $\emptyset$ we denote the empty family of subsets of $\mathbb{N}$. If $I$ is a downwards closed family of subsets of $\mathbb{N}$, let $I^{+}$stand for the family of subsets of $\mathbb{N}$ not in $I$, and let $I^{*}$ stand for the family of all complements in $\mathbb{N}$ of sets in $I$. Let us fix a bijection $\langle\cdot, \cdot\rangle: \mathbb{N} \times \mathbb{N} \rightarrow \mathbb{N}$. If $I$ and $J$ are downwards closed families of subsets of $\mathbb{N}$, let $I \ltimes J$ be the family of all $A \subseteq \mathbb{N}$ with

$$
\{n \in \mathbb{N}:\{m \in \mathbb{N}:\langle n, m\rangle \in A\} \notin J\} \in I .
$$

Since the bijection $\langle\cdot, \cdot\rangle$ is fixed, we will think of $I \ltimes J$ as a family of subsets of $\mathbb{N} \times \mathbb{N}$.

Note that, with the above notation, condition (3) in the definition of basic orders is simply $\left(\mathrm{Fin}^{+}, \mathcal{P}(\mathbb{N})\right)$-calibration.

Theorem 6.1. Let $D$ be basic and let $E$ be a metric separable space with a partial order. Let $H$ and $I$ be families of subsets of $\mathbb{N}$ with $H$ closed under taking supersets and subtracting finite sets. If $D \leq_{T} E$ and $E$ is $(H, I)$-calibrated, then $D$ is $(H, I)$ calibrated.

We start with a lemma.

Lemma 6.2. Let $D$ be basic and let $E$ be a separable metric space. Let $f: D \rightarrow E$. Then for any sequence $\left(x_{n}\right)$ in $D$ having an $H$-accumulation point there exists a sequence $\left(y_{n}\right)$ in $D$ such that $x_{n} \leq_{D} y_{n}$, for each $n$, and $\left(f\left(y_{n}\right)\right)$ has an $H$ accumulation point in $E$.

Proof. First, we prove a claim.

Claim. $\exists y \in E \forall U \ni y$ open $\exists A \in H \forall n \in A \exists p_{n} \in D\left(x_{n} \leq_{D} p_{n}\right.$ and $f\left(p_{n}\right) \in$ $U)$

Proof of claim. Assume that the claim fails, that is, for each $y \in E$ we have an open set $U_{y} \subseteq E$ containing $y$ with the following property:

(6.1) $\forall A \in H A \cap A_{y} \neq \emptyset$ where $A_{y}=\left\{n \in \mathbb{N}: \forall p \in D\left(x_{n} \leq_{D} p \Rightarrow f(p) \notin U_{y}\right)\right\}$

Since $E$ is separable, we can pick $y_{i} \in E, i \in \mathbb{N}$, so that

$$
E=\bigcup_{i \in \mathbb{N}} U_{y_{i}} \text {. }
$$

If $x$ is an $H$-accumulation point of $\left(x_{n}\right)$, then it follows from (6.1) that for each $i$, we can pick an increasing sequence $\left(n_{k}^{i}\right)_{k}$ such that $n_{k}^{i} \in A_{y_{i}}$ for all $k$ and $x_{n_{k}^{i}} \rightarrow x$ 
as $k \rightarrow \infty$. It follows now from Lemma 3.2 that there are $k_{i}$ with $\left\{x_{n_{k_{i}}}: i \in \mathbb{N}\right\}$ bounded, that is, for each $i$ we can choose an $n_{i} \in A_{y_{i}}$ such that $\left\{x_{n_{i}}: i \in \mathbb{N}\right\}$ is bounded. Let $p \in D$ be its bound. By the definition of the $A_{y}$ 's (6.1), for all $i \in \mathbb{N}$, $f(p) \notin U_{y_{i}}$ which contradicts (6.2), which finishes the proof of the claim.

Fix a $y \in E$ as in the claim. Let $U_{m}, m \in \mathbb{N}$, be a decreasing open basis at $y$ with $U_{0}=E$. Let $A_{m} \subseteq \mathbb{N}$ be such that $A_{m} \in H$ and there are $p_{n}^{m}, n \in A_{m}$, with $x_{n} \leq_{D} p_{n}^{m}$ and $f\left(p_{n}^{m}\right) \in U_{m}$. Since $U_{0}=E$, without loss of generality, we can assume that $A_{0}=\mathbb{N}$. Define

$$
y_{n}=p_{n}^{m} \text { for } n \in\left(\left(A_{m} \backslash \bigcup_{i>m} A_{i}\right) \cup\left(A_{m} \cap\{0, \ldots, m\}\right)\right) \backslash \bigcup_{i<m}\left(A_{i} \cap\{0, \ldots, i\}\right) \text {. }
$$

Note that, for all $n \in \mathbb{N}, y_{n}$ is defined, $x_{n} \leq_{D} y_{n}$ and that $y$ is an $H$-accumulation point of $\left(f\left(y_{n}\right)\right)$. This last condition follows from the facts that, for each $m$,

$$
A_{m} \backslash \bigcup_{i<m}\left(A_{i} \cap\{0, \ldots, i\}\right) \subseteq\left\{n \in \mathbb{N}: f\left(y_{n}\right) \in U_{m}\right\}
$$

and that $H$ is closed under taking supersets and subtracting finite sets.

Proof of Theorem 6.1. Let $f: D \rightarrow E$ be a Tukey reduction. Let $x_{n} \in D, n \in \mathbb{N}$, be a sequence having an $H$-accumulation point. We will show that there is $A \in H$ such that for any $B \in I,\left\{x_{n}: n \in A \cap B\right\}$ is bounded. By Lemma 6.2, there exists a sequence $\left(y_{n}\right)$ of elements of $D$ with $x_{n} \leq_{D} y_{n}$, for each $n$, and with $\left(f\left(y_{n}\right)\right)$ having an $H$-accumulation point. Since $E$ is $(H, I)$-calibrated, there exists $A \subseteq \mathbb{N}$ with $A \in H$ such that, for any $B \in I,\left\{f\left(y_{n}\right): n \in A \cap B\right\}$ is bounded. Since $f$ is Tukey, for any $B \in I,\left\{y_{n}: n \in A \cap B\right\}$, and, therefore, also $\left\{x_{n}: n \in A \cap B\right\}$, is bounded in $D$.

6.1. (Fin $\left.{ }^{*}, \mathcal{P}(\mathbb{N})\right)$-calibration. By taking $H=$ Fin $^{*}$ and $I=\mathcal{P}(\mathbb{N})$, we obtain, by Theorem 6.1 , a property which is preserved under Tukey reduction; the property can be stated as saying that for each converging sequence there is an element bounding all but finitely many elements of the sequence. In the lemma below, we show that this property holds for $\sigma$-ideals of compact sets (in fact, even relative $\sigma$-ideals) and fails for non-trivial analytic P-ideals. Therefore, no partial order from this latter class can be Tukey reduced to a partial order in the former one.

Proposition 6.3. $\quad$ (i) Each relative $\sigma$-ideal of compact sets is $\left(\right.$ Fin $\left.{ }^{*}, \mathcal{P}(\mathbb{N})\right)$ calibrated.

(ii) An analytic $P$-ideal is $\left(F i n^{*}, \mathcal{P}(\mathbb{N})\right)$-calibrated if, and only if, it is a trivial modification of Fin or is isomorphic to $\emptyset \ltimes$ Fin.

Proof. (i) If $I$ is a relative $\sigma$-ideal of compact sets and $\left(K_{n}\right)$ is a sequence of elements in $I$ converging to an element $K$ of $I$, then $K \cup \bigcup_{n} K_{n}$ is a compact set in $I$ bounding the sequence $\left(K_{n}\right)$.

(ii) Only the implication $\Rightarrow$ needs justification. Let $I=\operatorname{Exh}(\phi)$ for a lsc submeasure $\phi$. If there is an increasing sequence of natural numbers $\left(n_{k}\right)$ such that $\left\{n_{k}: k \in \mathbb{N}\right\} \notin I$ and $\lim _{k} \phi\left(\left\{n_{k}\right\}\right)=0$, then $\left\{n_{k}\right\} \rightarrow \emptyset$, as $k \rightarrow \infty$, in the submeasure topology on $I$ but, for all $K \in \mathbb{N},\left\{n_{k}: k \geq K\right\} \notin I$. Thus, $I$ is not 
$\left(\right.$ Fin $\left.^{*}, \mathcal{P}(\mathbb{N})\right)$-calibrated. We can, therefore, assume that such a sequence $\left(n_{k}\right)$ does not exist.

Let $x_{n}=\left\{k \in \mathbb{N}: \frac{1}{n} \geq \phi(\{k\})>\frac{1}{n+1}\right\}$ with the convention that $1 / 0=\infty$. Note that each element of $I$ intersects each $x_{n}$ in a finite set. Conversely, by our assumption from the preceding paragraph, if $y$ intersects each $x_{n}$ in a finite set, $y$ is in $I$. Thus, if only finitely many of the $x_{n}$ 's are infinite, then $I$ is a trivial modification of Fin. If infinitely many of the $x_{n}$ 's are infinite, by taking finite unions of them, we can partition $\mathbb{N}$ into infinite sets $y_{n}, n \in \mathbb{N}$, so that a set is in $I$ precisely when its intersection with each $y_{n}$ is finite. Thus, $I$ is isomorphic with $\emptyset \ltimes$ Fin.

It was proved by Fremlin [3, Proposition $3 \mathrm{M}(\mathrm{b})$ ] that the P-ideal $\mathcal{Z}_{0}$ is not Tukey below NWD. The following corollary generalizes this result.

Corollary 6.4. If $I$ is an analytic P-ideal and $J$ a relative $\sigma$-ideal of compact sets with $I \leq_{T} J$, then I is a trivial modification of Fin or is isomorphic to $\emptyset \ltimes$ Fin.

Proof. This is immediate from Theorem 6.1 and Proposition 6.3.

In the following proposition we record a simple observation on the bottom part of the Tukey order among $\left(\right.$ Fin $\left.^{*}, \mathcal{P}(\mathbb{N})\right)$-calibrated basic orders. It is a continuation of Proposition 4.3.

Proposition 6.5. Let $D$ be a $\left(\right.$ Fin $\left.^{*}, \mathcal{P}(\mathbb{N})\right)$-calibrated basic order.

(i) If $D$ is not locally compact, then $\mathbb{N}^{\mathbb{N}} \leq_{T} D$.

(ii) If $D$ is $\sigma$-compact non-compact, then $D \equiv_{T} \mathbb{N}$.

In particular, if $D$ is $\sigma$-compact, then it is locally compact.

Proof. To see (i), define $f$ as in Proposition 4.3(ii). Now, since $D$ is $\left(\right.$ Fin $\left.^{*}, \mathcal{P}(\mathbb{N})\right)$ calibrated, $f(D)$ is cofinal in $\mathbb{N}^{\mathbb{N}}$. To check it, let $\left(k_{n}\right)$ be an element of $\mathbb{N}^{\mathbb{N}}$. Then $\left(x_{k_{n}}^{n}\right)$ converges in $D$ to $z$. Since $D$ is $\left(\right.$ Fin $\left.^{*}, \mathcal{P}(\mathbb{N})\right)$-calibrated and directed, it follows that the sequence $\left(x_{k_{n}}^{n}\right)$ is bounded by an $x \in D$. Then $k_{n}<f(x)(n)$ for each $n \in \mathbb{N}$.

To see (ii), we need to show $\mathbb{N} \leq_{T} D$ and $D \leq_{T} \mathbb{N}$. Since $D$ is directed by condition (1) in the definition of basic orders, the first of these inequalities follows immediately from $D \mathbb{Z}_{T} 1$ which is a consequence of Proposition 4.3(i). To show the other inequality, it will suffice to see that there exists a countable subset of $D$ cofinal in $D$. We claim that, in fact, each $x \in D$ has a neighborhood which is bounded. Assume towards a contradiction that this is not the case, that is, that there is an $x_{0} \in D$ such that no open set containing $x_{0}$ is bounded. Fix an increasing sequence $L_{n}, n \in \mathbb{N}$, of compact subsets of $D$ whose union is $D$ and a decreasing sequence $U_{n}, n \in \mathbb{N}$, constituting a topological basis at $x_{0}$. By Lemma 4.4, for each $n$ there exists a finite set $F_{n} \subseteq U_{n}$ not bounded by any element of $L_{n}$. Then we can arrange $\bigcup_{n} F_{n}$ in a sequence converging to $x_{0}$. By assumption this sequence is bounded by some $z_{0} \in D$ which leads to a contradiction since $z_{0}$ cannot be an element of any $L_{n}$.

The "in particular" part, is immediate from (i) and (ii) and the fact that $\mathbb{N}^{\mathbb{N}} \mathbb{Z}_{T}$ $\mathbb{N}$. 
6.2. $\left((\text { Fin } \ltimes \text { Fin })^{+}, \emptyset \ltimes\right.$ Fin $)$-calibration. We take now $H=(\text { Fin } \ltimes \text { Fin })^{+}$and $I=\emptyset \ltimes$ Fin in Theorem 6.1 and obtain another invariant of Tukey reducibility. We will leave it to the reader to check the following easy proposition.

Proposition 6.6. If a basic order is (Fin*, $\mathcal{P}(\mathbb{N}))$-calibrated, then it is ( $(\mathrm{Fin} \ltimes$ Fin $)^{+}, \emptyset \ltimes$ Fin)-calibrated.

The following lemma gives a simple criterion for being $\left((\text { Fin } \ltimes \text { Fin })^{+}, \emptyset \ltimes\right.$ Fin)calibrated for an analytic P-ideal.

Lemma 6.7. Let $I=\operatorname{Exh}(\phi)$ be an analytic P-ideal with $\phi$ a lsc submeasure. The following conditions are equivalent:

(i) I is $\left((\text { Fin } \ltimes \text { Fin })^{+}, \emptyset \ltimes\right.$ Fin $)$-calibrated.

(ii) for any $\epsilon>0$ there exists $\delta>0$ such that any disjoint sequence $\left(x_{n}\right)$ of finite sets with $\sup _{n} \phi\left(x_{n}\right)<\delta$ has a subsequence $\left(x_{n_{k}}\right)$ with $\phi\left(\bigcup_{k} x_{n_{k}}\right)<\epsilon$.

Proof. To see $(\mathrm{i}) \Rightarrow\left(\right.$ ii), assume that (ii) fails. Then there exists an $\epsilon_{0}>0$ and disjoint sequences $\left(w_{n}^{i}\right)_{n}, i \in \mathbb{N}$, of finite sets such that

$$
\forall i, n \phi\left(w_{n}^{i}\right)<\frac{1}{i}
$$

and

$$
\forall i \forall a \subseteq \mathbb{N} \text { infinite } \phi\left(\bigcup_{n \in a} w_{n}^{i}\right)>\epsilon_{0} .
$$

By $(6.3), \emptyset$ is an $(\text { Fin } \ltimes \text { Fin })^{+}$-accumulation point of the sequence $\left(w_{n}^{i}\right)_{(i, n) \in \mathbb{N} \times \mathbb{N}}$. On the other hand, if $A \subseteq \mathbb{N} \times \mathbb{N}$ is in $(\text { Fin } \ltimes \text { Fin })^{+}$, for infinitely many $n, A \cap(\{i\} \times \mathbb{N})$ is infinite. Thus, (6.4) allows us to pick $f \in \mathbb{N}^{\mathbb{N}}$ such that $\bigcup\left\{w_{n}^{i}:(n, i) \in A\right.$ and $n \leq$ $f(i)\} \notin I$, that is, $\left\{w_{n}^{i}:(n, i) \in A\right.$ and $\left.n \leq f(i)\right\}$ is not bounded.

We prove now (ii) $\Rightarrow(\mathrm{i})$. Let $\left(x_{n}^{i}\right)_{(i, n) \in \mathbb{N} \times \mathbb{N}}$ be a sequence of elements of $I$ with an $(\text { Fin } \ltimes \text { Fin })^{+}$-accumulation point $x \in I$. It will be enough to show that we can pass to a subsequence (with respect to the index $i$ ) of $\left(\left(x_{n}^{i}\right)_{n}\right)_{i}$ and then, for each value of the index $i$ in the chosen subsequence, we can go to a subsequence (with respect to the index $n$ ) of $\left(x_{n}^{i}\right)_{n}$ so that the resulting double sequence, denoted again by $\left(x_{n}^{i}\right)_{(i, n) \in \mathbb{N} \times \mathbb{N}}$, has union in $I$, that is, is bounded in $I$.

By considering $\left(x_{n}^{i} \triangle x\right)_{(i, n) \in \mathbb{N} \times \mathbb{N}}$, we can assume that $x=\emptyset$. Our assumption, condition (ii), guarantees that for $\epsilon_{i}=2^{-i}$ there is $\delta_{i}>0$ such that the rest of the condition holds. By going to a subsequence (with respect to the index $i$ ) of the sequence of sequences $\left(\left(x_{n}^{i}\right)_{n}\right)_{i}$ and then, for each $i$, to a subsequence of $\left(x_{n}^{i}\right)_{n}$ we can assume that, for each $i$ and $n$,

$$
\phi\left(x_{n}^{i}\right)<\min \left(\frac{\delta_{i}}{2}, 2^{-i}\right) .
$$

Fix $i$. We can pass to a subsequence (with respect to the index $n$ ) and assume that $\left(x_{n}^{i}\right)_{n}$ converges pointwise to some $y^{i} \subseteq \mathbb{N}$. Note that

$$
\phi\left(y^{i}\right) \leq 2^{-i} .
$$


It is not difficult to go to a further subsequence of $\left(x_{n}^{i}\right)_{n}$ and find an increasing sequence of natural numbers $m_{n}^{i}$ so that for all $n$

$$
\left(x_{n}^{i} \backslash y^{i}\right) \cap m_{n}^{i}=\emptyset
$$

and

$$
\phi\left(x_{n}^{i} \backslash m_{n+1}^{i}\right)<2^{-i-n} .
$$

Let

$$
w_{n}^{i}=\left(x_{n}^{i} \backslash y^{i}\right) \cap m_{n+1}^{i} .
$$

By (6.5) and (6.7), $\left(w_{n}^{i}\right)_{n}$ is a sequence of disjoint finite sets with $\sup _{n} \phi\left(w_{n}^{i}\right)<\delta_{i}$. Thus, by our assumption (ii), we can go to a subsequence of $\left(w_{n}^{i}\right)_{n}$ to guarantee

$$
\phi\left(\bigcup_{n} w_{n}^{i}\right)<2^{-i} \text {. }
$$

Note now that (6.9) implies that, for any $f \in \mathbb{N}^{\mathbb{N}}, \bigcup\left\{w_{n}^{i}: n \leq f(i)\right\} \in I$. From this, the definition of the $w_{n}^{i}$ 's, and (6.8), it follows that $\bigcup\left\{x_{n}^{i} \backslash y^{i}: n \leq f(i)\right\} \in I$. This, in turn, in conjunction with (6.6) gives $\bigcup\left\{x_{n}^{i}: n \leq f(i)\right\} \in I$, and we are done.

After [2] we call an ideal $I$ a generalized density $P$-ideal if there exist submeasures $\phi_{i}, i \in \mathbb{N}$, on $\mathbb{N}$ with finite and pairwise disjoint supports such that $I=\operatorname{Exh}(\phi)$ where $\phi(x)=\sup _{i} \phi_{i}(x)$ for $x \subseteq \mathbb{N}$ or, in other words,

$$
I=\left\{x \subseteq \mathbb{N}: \inf _{n} \sup _{i \geq n} \phi_{i}(x)=0\right\} .
$$

(A finite set $F \subseteq \mathbb{N}$ is the support of a submeasure $\phi_{i}$ if $\phi_{i}(\mathbb{N} \backslash F)=0$ and $\phi_{i}(\{n\})>0$ for each $n \in F$.) It was noticed in [2] that $\mathcal{Z}_{0}$ is a generalized density P-ideal. Moreover, all ideals $I_{S}$, for $S \subseteq \mathbb{N}$, studied in [8, Theorem 6] are by their very definition generalized density ideals.

Proposition 6.8. $\quad$ (i) Each generalized density P-ideal is $\left((\text { Fin } \ltimes \text { Fin })^{+}, \emptyset \ltimes\right.$ Fin)-calibrated.

(ii) Let $I$ be an $F_{\sigma}$ P-ideal. Then I is $\left((F i n \ltimes F i n)^{+}, \emptyset \ltimes\right.$ Fin $)$-calibrated if, and only if, $I$ is a trivial modification of Fin.

Proof. (i) Each generalized density P-ideal easily fulfills condition (ii) of Lemma 6.7. In fact, given $\epsilon>0$ the conclusion of this condition holds for any positive $\delta<\epsilon$.

(ii) The implication $\Leftarrow$ is clear. To see $\Rightarrow$, we will show that an $F_{\sigma}$ P-ideal $I$ which is not a trivial modification of Fin does not fulfill condition (ii) from Lemma 6.7. Let $I=\operatorname{Exh}(\phi)$. Since $I$ is $F_{\sigma}$, by the proof of [9, Theorem 3.4], there exists an $\epsilon>0$ such that if $\left(x_{n}\right)$ is a disjoint sequence of finite sets with $\inf _{n} \phi\left(x_{n}\right)>0$, then $\phi\left(\bigcup_{n} x_{n}\right)>\epsilon$. Since $I$ is not a trivial modification of Fin, for any given $\delta>0,\{n: \phi(\{n\})<\delta / 2\} \notin I$. Now by splitting this set into appropriate disjoint intervals, we obtain a disjoint sequence $\left(x_{n}\right)$ of finite sets such that

$$
\frac{\delta}{2}<\inf _{n} \phi\left(x_{n}\right) \leq \sup _{n} \phi\left(x_{n}\right)<\delta .
$$


Then for any subsequence $\left(x_{n_{k}}\right)$ of $\left(x_{n}\right)$ we have $\phi\left(\bigcup_{k} x_{n_{k}}\right)>\epsilon$, which finishes the proof of the proposition.

It was proved in [8, Theorem 7] that $\mathcal{I}_{1 / n} \mathbb{Z}_{T} \mathcal{Z}_{0}$. This is a very special case of the corollary below.

Corollary 6.9. If $I$ is an $F_{\sigma} P$-ideal, $J$ is a generalized density P-ideal and $I \leq_{T} J$, then $I$ is a trivial modification of Fin.

Proof. This is an immediate consequence of Proposition 6.8 and Theorem 6.1.

\section{Analytic P-ideals and Relative $\sigma$-ideals of CompaCt Sets}

As was shown in Corollary 6.4 no non-trivial analytic $P$-ideal is Tukey reducible to a relative $\sigma$-ideal of compact sets. However, it turns out that there exists a functor associating in a canonical way with each analytic P-ideal a relative $\sigma$-ideal of compact subsets of $2^{\mathbb{N}}$. In the present section, we define and study this functor.

A compact subset $K$ of $2^{\mathbb{N}}$ is called monotone if, for any $x \in K$ and $y \subseteq x$, $y \in K$. We will associate an ideal of compact monotone subsets of $2^{\mathbb{N}}$ with each ideal of subsets of $\mathbb{N}$. Let $I$ be a ideal of subsets of $\mathbb{N}$. Define

$$
D(I)=\left\{K \subseteq 2^{\mathbb{N}}: K \text { compact, monotone and } \exists x \in I \forall n x \backslash\{0, \ldots, n\} \notin K\right\} .
$$

The family of monotone compact sets $D(I)$ comes up naturally and its use is instrumental in the proof of the theorem characterizing analytic P-ideals as those of the form $\operatorname{Exh}(\phi)$ for a lsc submeasure $\phi[9]$.

It is easy to see that the union of two elements of $D(I)$ is in $D(I)$ and that a monotone compact subset of an element of $D(I)$ is in $D(I)$. It is straightforward to check that if $I$ is an analytic P-ideal, then $D(I)$ is a $\sigma$-ideal of monotone compact sets, that is, in addition to the two properties stated in the previous sentence, we also have that a compact set which is a countable union of elements of $D(I)$ is in $D(I)$. Thus, since monotone compact sets form a closed family of compact sets, $D(I)$ is a relative $\sigma$-ideal of compact sets. We consider $D(I)$ equipped with inclusion as partial order.

First we describe an ordering which has a combinatorial definition and which, as is proved in the next lemma, is Tukey equivalent to $D(I)$. For an ideal $I$ of subsets of $\mathbb{N}$ different from Fin, define

$$
D^{\prime}(I)=\left\{\left(x_{n}\right): \forall n x_{n} \subseteq \mathbb{N} \text { is finite and non-empty, } x_{n}<x_{n+1} \text {, and } \bigcup_{n} x_{n} \in I\right\}
$$

where, for $x, y \subseteq \mathbb{N}$, we write $x<y$ if each element of $x$ is smaller than each element of $y$.

We order $D^{\prime}(I)$ by $\leq_{D^{\prime}(I)}$ defined by

$$
\left(x_{n}\right) \leq_{D^{\prime}(I)}\left(y_{n}\right) \Leftrightarrow \forall n \exists m x_{m} \subseteq y_{n} .
$$

It is easy to check that $\leq_{D^{\prime}(I)}$ is a directed partial order.

Lemma 7.1. If I is an ideal of subsets of $\mathbb{N}$ different from Fin, then $(D(I), \subseteq) \equiv_{T}$ $\left(D^{\prime}(I), \leq_{D^{\prime}(I)}\right)$ 
Proof. Since $D(I)$ consists of compact and monotone sets, a set $K$ is in $D(I)$ precisely when for some $\left(x_{n}\right) \in D^{\prime}(I)$,

$$
K \subseteq\left\{y \subseteq \mathbb{N}: \forall n x_{n} \nsubseteq \subseteq\right\} .
$$

Define for $\left(x_{n}\right) \in D^{\prime}(I)$,

$$
F\left(\left(x_{n}\right)\right)=\left\{y \subseteq \mathbb{N}: \forall n x_{n} \not \subset y\right\} .
$$

By what was said above $F$ defines a function from $D^{\prime}(I)$ to $D(I)$ with cofinal range. It is a straightforward consequence of the definition of $\leq_{D^{\prime}(I)}$ that $F$ is a monotone map. Thus, $D(I) \leq_{T} D^{\prime}(I)$. Note, however, that $F$ is also a Tukey map. Indeed, if $K \in D(I)$ and $\left(y_{n}\right) \in D^{\prime}(I)$ is such that for any $z \in K$, for all $n \in \mathbb{N}, y_{n} \not \subset z$, then the set $\left\{\left(x_{n}\right) \in D^{\prime}(I): F\left(\left(x_{n}\right)\right) \subseteq K\right\}$ is $\leq_{D^{\prime}(I)}$-bounded by $\left(y_{n}\right)$.

In this section, we prove several results describing the behavior of the operator $D(I)$ for analytic P-ideals $I$. As the computations following the proof of Theorem 7.6 show, the various non-triviality assumptions on analytic P-ideals in these results are necessary.

The first theorem states that the operator $D$ respects the Tukey order. Since $I \leq_{T} \mathcal{I}_{1 / n}$ for any analytic P-ideal $I$, an immediate consequence of this theorem is the fact that $D\left(\mathcal{I}_{1 / n}\right)$ is the Tukey top element among all basic orders of the form $D(I)$ for analytic P-ideals $I$.

Theorem 7.2. Let $I$ and $J$ be analytic P-ideals with $J \neq F$ in. If $I \leq_{T} J$, then $D(I) \leq_{T} D(J)$.

To establish the above theorem, we will need a result giving a combinatorial condition on $I$ and $J$ which implies $D(I) \leq_{T} D(J)$. This is included in the following lemma. Note that, for arbitrary ideals (and not only analytic P-ideals) $I$ and $J$, this lemma immediately yields that if $I$ is Rudin-Blass below $J$, then $D(I) \leq_{T} D(J)$.

In the lemma below and in the proof of Theorem 7.2, for a sequence $\left(x_{n}\right)$ of finite subsets of $\mathbb{N}$, we write $x_{n} \rightarrow \emptyset$ if each natural number belongs to finitely many $x_{n}$ 's. It should not be confused with the usually stronger condition that $\left(x_{n}\right)$ converges to $\emptyset$ in the submeasure topology of some analytic P-ideal.

Lemma 7.3. Let $I, J$ be ideals of subsets of $\mathbb{N}$ with $J \neq F$ in. Assume there exists $h:$ Fin $\rightarrow$ Fin such that for any sequence $\left(x_{n}\right)$ of elements of Fin, we have

(i) if $x_{n} \rightarrow \emptyset$ and $\bigcup_{n} x_{n} \in I$, then there exists a subsequence $\left(x_{k_{n}}\right)$ of $\left(x_{n}\right)$ such that $h\left(x_{k_{n}}\right) \rightarrow \emptyset$ and $\bigcup_{n} h\left(x_{k_{n}}\right) \in J$;

(ii) if $h\left(x_{n}\right) \rightarrow \emptyset$ and $\bigcup_{n} h\left(x_{n}\right) \in J$, then $\bigcup_{n} x_{n} \in I$.

Then $D(I) \leq_{T} D(J)$.

Proof. If $I=$ Fin, the conclusion is clear since $D($ Fin $) \equiv_{T} 1$ by definition of $D$. Thus, assume that both $I \neq$ Fin and $J \neq$ Fin. In light of Lemma 7.1, it will suffice to prove the conclusion of the lemma for $D^{\prime}(I)$ and $D^{\prime}(J)$.

We now modify $h$ to $h^{\prime}:$ Fin $\rightarrow$ Fin so that $h^{\prime}$ fulfills (i) and the following strengthening of (ii):

(ii') if $h^{\prime}\left(x_{n}\right) \rightarrow \emptyset$ and $\bigcup_{n} h^{\prime}\left(x_{n}\right) \in J$, then $x_{n} \rightarrow \emptyset$ and $\bigcup_{n} x_{n} \in I$. 
To define $h^{\prime}$ fix an infinite set $a \in J$. Let

$$
h^{\prime}(x)=h(x) \cup\{\min \{k \in a: \min x \leq k\}\}
$$

where $\min \emptyset=0$. The first part of the conclusion of (i) holds for $h^{\prime}$ since $x_{n} \rightarrow \emptyset \mathrm{im}$ plies $\min \{k \in a: \min x \leq k\} \rightarrow \infty$ while the second part holds since $\bigcup_{n} h^{\prime}\left(x_{k_{n}}\right) \subseteq$ $a \cup \bigcup_{n} h\left(x_{k_{n}}\right)$. Point (ii') holds since $h^{\prime}\left(x_{n}\right) \rightarrow \emptyset$ and $\bigcup_{n} h^{\prime}\left(x_{n}\right) \in J$ imply the analogous properties for the sequence $\left(h\left(x_{n}\right)\right)_{n}$ and, therefore, also $\bigcup_{n} x_{n} \in I$. By the very definition of $h^{\prime}, h^{\prime}\left(x_{n}\right) \rightarrow \emptyset$ gives $\min \left\{k \in a: \min x_{n} \leq k\right\} \rightarrow \infty$ as $n \rightarrow \infty$, hence $x_{n} \rightarrow \emptyset$. Additionally, note that $h^{\prime}(x) \neq \emptyset$ for all $x \in$ Fin.

Now we modify $h^{\prime}$ to $h^{\prime \prime}:$ Fin $\rightarrow$ Fin so that $h^{\prime \prime}$ fulfills (i) and (ii') and, additionally, is one-to-one. Fix again $a \in J$ infinite. Note that if for each $x \in$ Fin we have $h^{\prime}(x) \subseteq h^{\prime \prime}(x)$ and $h^{\prime \prime}(x) \backslash h^{\prime}(x) \subseteq\left\{k \in a: k>\max h^{\prime}(x)\right\}$, then $h^{\prime \prime}$ still fulfills (i) and (ii'). (To see the first part of (i) use $h^{\prime}\left(x_{k_{n}}\right) \neq \emptyset$ for all $n$.) Let $x_{p}$, $p \in \mathbb{N}$, be a list of Fin in which each element appears once. Define, by induction on $p, h^{\prime \prime}\left(x_{0}\right)=h^{\prime}\left(x_{0}\right)$ and

$$
h^{\prime \prime}\left(x_{p+1}\right)=h^{\prime}\left(x_{p}\right) \cup\{m\}
$$

where $m$ is the smallest element of $a$ strictly bigger that the numbers $\max h^{\prime}\left(x_{p+1}\right)$ and $\max h^{\prime \prime}\left(x_{i}\right)$ for $i \leq p$. Clearly $h^{\prime \prime}$ is one-to-one and, by what was said above, fulfills (i) and (ii').

Thus, we can assume that the function $h$ has these properties. Let $\left(x_{n}\right) \in D^{\prime}(I)$. Then $x_{n} \rightarrow \emptyset$ and $\bigcup_{n} x_{n} \in I$. Hence, by (i), there is a subsequence $\left(x_{k_{n}}\right)$ such that $\bigcup_{n} h\left(x_{k_{n}}\right) \in J$ and $h\left(x_{k_{n}}\right) \rightarrow \emptyset$. This last condition allows us to go to a further subsequence to insure $h\left(x_{k_{n}}\right)<h\left(x_{k_{n+1}}\right)$ for all $n$. It follows that $\left(h\left(x_{k_{n}}\right)\right) \in D^{\prime}(J)$. Define $f\left(\left(x_{n}\right)\right)=\left(h\left(x_{k_{n}}\right)\right)$. We claim that $f$ is a Tukey reduction of $D^{\prime}(I)$ to $D^{\prime}(J)$.

Fix $\left(y_{n}\right) \in D^{\prime}(J)$. Since $h$ is one-to-one, for each $n$, the set $\left\{x \in\right.$ Fin $\left.: h(x) \subseteq y_{n}\right\}$ is finite. Moreover, since $\left(y_{n}\right) \in D^{\prime}(J), y_{n} \rightarrow \emptyset$ and $\bigcup_{n} y_{n} \in J$. Thus, by (ii'), a sequence enumerating $\left\{x \in\right.$ Fin $\left.: \exists n h(x) \subseteq y_{n}\right\}$ tends to $\emptyset$ and the union of this sequence is in $I$. It follows that, if we let $z_{n}^{\prime}=\bigcup\left\{x \in\right.$ Fin $\left.: h(x) \subseteq y_{n}\right\}$, then each $z_{n}^{\prime}$ is finite, $z_{n}^{\prime} \rightarrow \emptyset$, and $\bigcup_{n} z_{n}^{\prime} \in I$. Let $\left(z_{n}\right)$ be a subsequence of $\left(z_{n}^{\prime}\right)$ such that $z_{n}<z_{n+1}$ for all $n$. Then $\left(z_{n}\right) \in D^{\prime}(I)$. We prove that $\left(z_{n}\right)$ is a bound on the set $\left\{\left(x_{n}\right) \in D^{\prime}(I): f\left(\left(x_{n}\right)\right) \leq_{D^{\prime}(J)}\left(y_{n}\right)\right\}$. If $\left(x_{n}\right)$ is in this set, then it follows from the definition of $f$ that for each $n$ there is a $k$ such that $h\left(x_{k}\right) \subseteq y_{n}$. This immediately implies, by the definition of $\left(z_{n}\right)$, that for each $n$ there exists a $k$ with $x_{k} \subseteq z_{n}$. Thus, we proved that $D^{\prime}(I) \leq_{T} D^{\prime}(J)$.

Proof of Theorem 7.2. We show that if $I \leq_{T} J$, then there exists a function $h$ as in Lemma 7.3.

Let $\phi$ and $\psi$ be lsc submeasures with $I=\operatorname{Exh}(\phi)$ and $J=\operatorname{Exh}(\psi)$. By modifying $\psi$ to $\psi^{\prime}(x)=\psi(x)+\sum_{i \in x} 2^{-i}$, we get a lsc submeasure $\psi^{\prime}$ such that $J=\operatorname{Exh}\left(\psi^{\prime}\right)$, $\psi^{\prime}(x)>0$ for $x \neq \emptyset$. Thus, we can assume that $\psi$ itself has this property. Below we consider $I$ and $J$ with the submeasure topologies.

Let $f: I \rightarrow J$ be a Tukey map. By Theorem 5.3(i), we can assume that $f$ is measurable with respect to the $\sigma$-algebra generated by analytic sets, in particular, $f$ is Baire measurable. Since $I$ is Polish, there is a comeager subset such that 
the restriction of $f$ to it is continuous [6,8.38]. Thus, there exists a dense set $Q \subseteq I$ and $a \in Q$ such that $f \mid Q$ is continuous at $a$. Note now that the function $I \ni x \rightarrow f(x \triangle a) \triangle f(a) \in J$ is also a Tukey map. By redefining $f$ to be this map, we can assume that $f(\emptyset)=\emptyset$ and that there is a dense set $Q \subseteq I$ such that $\emptyset \in Q$ and $f \mid Q$ is continuous at $\emptyset$.

Now let $x \subseteq \mathbb{N}$ be finite. Since $Q$ is dense, we can chose $q_{x} \in Q$ so that, with the usual convention $\max \emptyset=0$, we have

$$
x \subseteq q_{x} \text { and } \phi\left(x \triangle q_{x}\right)<2^{-\max x} .
$$

We can, and will, assume that $q_{\emptyset}=\emptyset$. We now define $h:$ Fin $\rightarrow$ Fin by letting $h(x)$ be a finite set such that

$$
\psi\left(h(x) \triangle f\left(q_{x}\right)\right)<2^{-2 \max x} .
$$

We claim that $h$ fulfills conditions (i) and (ii) of Lemma 7.3.

Let $\left(x_{n}\right)$ be a sequence of elements of Fin. To see (i), let $x_{n} \rightarrow \emptyset$ and $\bigcup_{n} x_{n} \in I$. Then by the second part of $(7.1), \lim _{n} \phi\left(q_{x_{n}}\right)=0$. By continuity of $f \mid Q$ at $\emptyset$, $\lim _{n} \psi\left(f\left(q_{x_{n}}\right)\right)=0$. By (7.2), this implies that $\lim _{n} \psi\left(h\left(x_{n}\right)\right)=0$. By going to a subsequence $\left(x_{k_{n}}\right)$, we can insure that $\sum_{n} \psi\left(h\left(x_{k_{n}}\right)\right)<\infty$ whence $h\left(x_{k_{n}}\right) \rightarrow \emptyset$ and $\bigcup_{n} h\left(x_{k_{n}}\right) \in J$. To see (ii), assume that $h\left(x_{n}\right) \rightarrow \emptyset$ and $\bigcup_{n} h\left(x_{n}\right) \in J$ for a sequence $\left(x_{n}\right)$ of finite sets. If the sequence $\left(x_{n}\right)$ attains only finitely many values, then $\bigcup_{n} f\left(q_{x_{n}}\right) \in J$. Otherwise, we can assume that $\left(x_{n}\right)$ attains each value only once. Thus, $\sum_{n} 2^{-2 \max x_{n}}<\infty$ which together with our assumption and (7.2) gives $\bigcup_{n} f\left(q_{x_{n}}\right) \in J$. In either case, since $f$ is Tukey, we get that $\bigcup_{n} q_{x_{n}} \in I$. Now, the first part of (7.1) gives that $\bigcup_{n} x_{n} \in I$, and we are done.

The next theorem shows that the operator $D$ lowers Tukey complexity.

Theorem 7.4. If I is an analytic P-ideal which is not a trivial modification of Fin, then $D(I) \leq_{T} I$. If, additionally, $I$ is not isomorphic to $\emptyset \ltimes$ Fin, then $D(I)<_{T} I$.

We will need a lemma.

Lemma 7.5. Let $I$ be an ideal of subsets of $\mathbb{N}$. Then $D(I) \leq_{T} I \times \mathbb{N}^{\mathbb{N}}$.

Proof. If $I=$ Fin, $D(I)$ consists only of the empty set and the conclusion is clear. If $I \neq$ Fin, in view of Lemma 7.1, it suffices to show that $D^{\prime}(I) \leq_{T} I \times \mathbb{N}^{\mathbb{N}}$. We $\operatorname{map}\left(x_{n}\right) \in D^{\prime}(I)$ to $\left(\bigcup_{n} x_{n},\left(\max x_{n}\right)_{n}\right) \in I \times \mathbb{N}^{\mathbb{N}}$. Now if $x \in I$ and $f \in \mathbb{N}^{\mathbb{N}}$ are given, consider the set

$$
A=\left\{\left(x_{n}\right) \in D^{\prime}(I): \bigcup_{n} x_{n} \subseteq x \text { and } \forall n \max x_{n} \leq f(n)\right\} .
$$

To see that this set is bounded in $D^{\prime}(I)$, it will suffice to show that given $k$ there is an $m$ such that for each $\left(x_{n}\right) \in A, x \cap[k, m) \supseteq x_{n}$ for some $n$. Note that for each $\left(x_{n}\right) \in D^{\prime}(I), k \leq \min x_{k}$, and for each $\left(x_{n}\right) \in A$, $\max x_{k} \leq f(k)$, so we can take $m$ to be $f(k)+1$.

Proof of Theorem 7.4. If $I$ is not a trivial modification of Fin, then, by [12], $\mathbb{N}^{\mathbb{N}} \leq_{T}$ $I$ and, therefore, $I \times \mathbb{N}^{\mathbb{N}} \leq_{T} I$. Thus, from Lemma 7.5, we get $D(I) \leq_{T} I$. If, additionally, $I$ is not isomorphic to $\emptyset \ltimes$ Fin, then $I \not_{T} D(I)$ by Corollary 6.4. 
The following theorem contains general upper and lower bounds for $D(I)$.

Theorem 7.6. Let $I$ be an analytic P-ideal. Then $D(I) \leq_{T} \mathrm{NWD}$ and, if I is not equal to Fin, $\mathbb{N}^{\mathbb{N}} \leq_{T} D(I)$.

Proof. Fix a lsc submeasure $\phi$ with $I=\operatorname{Exh}(\phi)$. Since the Polish submeasure topology $\tau$ on $I$ is stronger than the topology inherited by $I$ from $2^{\mathbb{N}}$, we conclude that if $K$ is a closed subset of $2^{\mathbb{N}}$, then $K \cap I$ is closed in $\tau$.

Claim. Let $K$ be compact monotone. Then $K \in D(I)$ if and only if $K \cap I$ is nowhere dense in $\tau$.

Proof of claim. By what was just said $K \cap I$ is $\tau$-closed. If it were not nowhere dense, we would be able to find $x_{0} \in I$ and $\epsilon>0$ such that $\left\{y \in I: \phi\left(y \triangle x_{0}\right)<\epsilon\right\} \subseteq$ $K$. Let $y_{0} \in I$ be such that $y_{0} \backslash\{0, \ldots, n\} \notin K$ for each $n$. By lower semicontinuity of $\phi$ we can find $n_{0}$ such that

$$
\phi\left(\left(\left(x_{0} \cap\left\{0, \ldots, n_{0}\right\}\right) \cup\left(y_{0} \cap\left\{n_{0}+1, n_{0}+2, \ldots\right\}\right)\right) \triangle x_{0}\right)<\epsilon .
$$

This means that $\left(x_{0} \cap\left\{0, \ldots, n_{0}\right\}\right) \cup\left(y_{0} \cap\left\{n_{0}+1, n_{0}+2, \ldots\right\}\right) \in K$ which implies, by monotonicity of $K$, that $y_{0} \cap\left\{n_{0}+1, n_{0}+2, \ldots\right\} \in K$ contradicting the choice of $y_{0}$.

Now let $K$ be compact monotone and such that $K \cap I$ is nowhere dense in $\tau$. This allows us to find $x_{k} \in I, k \in \mathbb{N}$, with $x_{k} \notin K$ and $\phi\left(x_{k}\right)<2^{-k}$. Then, by lower semicontinuity of $\phi, \bigcup_{k} x_{k} \in I$. For each $n, \bigcup_{k} x_{k} \backslash\{0, \ldots, n\}$ contains one of the $x_{k}$ 's which implies, by monotonicity of $K$, that it is not an element of $K$. Thus, $K \in D(I)$.

By the claim we have the following mapping

$$
D(I) \ni K \rightarrow K \cap I \in \mathrm{NWD}(I, \tau) .
$$

We show that it is a Tukey map. Fix $F \subseteq I$ which is closed and nowhere dense in $I$ with $\tau$. By the claim it will suffice to prove that

$$
\overline{\bigcup\{K \in D(I): K \cap I \subseteq F\}} \cap I,
$$

where the closure is taken in $2^{\mathbb{N}}$, is nowhere dense in $\tau$. We will show that this set is included in $F$. Note that $\bigcup\{K \in D(I): K \cap I \subseteq F\}$ is monotone. Therefore, we will be done if we show that

$$
\bar{A} \cap I=\overline{A \cap I}^{\tau}
$$

for any monotone set $A$. The inclusion $\supseteq$ is obvious. Let now $x \in \bar{A} \cap I$. Fix $x_{k} \rightarrow x$, as $k \rightarrow \infty$, with $x_{k} \in A$. Then, $x_{k} \cap x \rightarrow x$ and, since $x \in I$ and $A$ is monotone, $x_{k} \cap x \in A \cap I$. By the lower semicontinuity of $\phi, \phi\left(\left(x_{k} \cap x\right) \triangle x\right) \rightarrow 0$ as $k \rightarrow \infty$; thus, $x \in \overline{A \cap I}^{\tau}$.

It remains to show that $\mathbb{N}^{\mathbb{N}} \leq D(I)$ if $I$ is not Fin. Since $I$ is a P-ideal, $D(I)$ is a relative $\sigma$-ideal of compact sets. Thus, by Proposition 6.5(i), it will suffice to see that $D(I)$ with the Vietoris topology is not locally compact. Let $x \in I$ be infinite. Then $\mathcal{P}(x) \backslash$ Fin is not locally compact with the topology from $2^{\mathbb{N}}$. Now, by simple arguments involving definitions of topologies on $2^{\mathbb{N}}$ and $\mathcal{K}\left(2^{\mathbb{N}}\right)$, we get that the function

$$
\mathcal{P}(x) \backslash \text { Fin } \ni y \rightarrow \mathcal{P}(\mathbb{N} \backslash y) \in D(I)
$$


is a homeomorphic embedding of $\mathcal{P}(x) \backslash$ Fin onto a closed subset of $D(I)$. Thus, $D(I)$ is not locally compact either.

We can now compute the Tukey degree of $D(I)$ for simple analytic P-ideals. Directly from the definition $D($ Fin $)=\{\emptyset\}$, so we get

$$
D(\text { Fin }) \equiv_{T} 1 \text {. }
$$

Next we have

$D(I) \equiv_{T} \mathbb{N}^{\mathbb{N}}$ if $I$ is a trivial modification of Fin without being equal to Fin.

Indeed, in this situation $I \leq_{T} \mathbb{N}^{\mathbb{N}}$ and we get the above Tukey equivalence from Lemma 7.5 and Theorem 7.6. Since $\emptyset \ltimes$ Fin $\equiv_{T} \mathbb{N}^{\mathbb{N}}$, again from Lemma 7.5 and Theorem 7.6 , we get

$$
D(\emptyset \ltimes \operatorname{Fin}) \equiv_{T} \mathbb{N}^{\mathbb{N}} .
$$

The following theorem shows that the functor $I \rightarrow D(I)$ is not trivial, that is, it does not map all analytic P-ideals not equal Fin to $\mathbb{N}^{\mathbb{N}}$. As is customary, we denote by $\mathcal{E}_{\mu}$ the set of compact Lebesgue measure zero subsets of $2^{\mathbb{N}}$ ordered by inclusion. By a result of Fremlin [3], $\mathbb{N}^{\mathbb{N}}<_{T} \mathcal{E}_{\mu}$.

Theorem 7.7. $\mathcal{E}_{\mu} \leq_{T} D\left(\mathcal{I}_{1 / n}\right)$

Proof. By Lemma 7.1, Theorem 7.2, and the remark preceding this theorem, it will be enough to show that $\mathcal{E}_{\mu} \leq_{T} D^{\prime}(I)$ for some analytic P-ideal $I$. Let $I$ be an ideal of the form

$$
\left\{x \subseteq \mathbb{N}: \sum\left\{b_{n}: n \in x\right\}<\infty\right\},
$$

where $\left(b_{n}\right)$ is a sequence of positive reals converging to 0 whose sum is infinite and which is such that for each $n$ there is an arbitrarily large $m$ such that for some $k$

$$
b_{n}=\sum_{i=m}^{m+k} b_{i} .
$$

Clearly $I$ is an analytic P-ideal. The above condition on the $b_{n}$ 's allows us to pick a sequence of finite sets $x_{n} \subseteq \mathbb{N}, n \in \mathbb{N}$, and $y_{k}^{n} \subseteq x_{n+1}$ for $k \in x_{n}$ so that

(a) $x_{n}<x_{n+1}$ for all $n \in \mathbb{N}$;

(b) $b_{k}=\sum_{i \in y_{k}^{n}} b_{i}$ for $k \in x_{n}$;

(c) $\sum_{i \in x_{0}} b_{i} \geq 1$;

(d) $y_{k}^{n}$ for $k \in x_{n}$ are pairwise disjoint.

Define

$$
K=\left\{\left(m_{n}\right) \in \mathbb{N}^{\mathbb{N}}: m_{0} \in x_{0} \text { and } \forall n m_{n+1} \in y_{m_{n}}^{n}\right\} .
$$

Obviously, $K$ is compact. We equip $K$ with a finite Borel measure $\mu$ which is defined by assigning to each basic open set of the form

$$
\left\{\left(m_{n}\right) \in K: m_{0}=\bar{m}_{0}, \ldots, m_{N}=\bar{m}_{N}\right\},
$$

where $\bar{m}_{0} \in x_{0}$ and $\bar{m}_{n+1} \in y_{\bar{m}_{n}}^{n}$ for $n<N$, the value $b_{\bar{m}_{N}}$. Note that conditions (c) and (b) insure that $\mu$ is well-defined with $1 \leq \mu(K)<\infty$. Define now a function $F$ on $D^{\prime}(I)$ by letting

$$
F\left(\left(z_{n}\right)\right)=\left\{\left(m_{n}\right) \in K:\left(\left\{m_{n}\right\}\right) \leq_{D^{\prime}(I)}\left(z_{n}\right)\right\} .
$$


We claim that $F$ is a monotone map from $D^{\prime}(I)$ to $\mathcal{E}_{\mu}$ with cofinal range. This will show that $D(I) \leq_{T} \mathcal{E}_{\mu}$. First we show that the map goes into $\mathcal{E}_{\mu}$. The set $F\left(\left(z_{n}\right)\right)$ is closed since the relation $\leq_{D^{\prime}(I)}$ is closed in the topology on $D^{\prime}(I)$ which is inherited from the inclusion $D^{\prime}(I) \subseteq \operatorname{Fin}^{\mathbb{N}}$ where $\operatorname{Fin}^{\mathbb{N}}$ is equipped with the product topology. To see that $\mu\left(F\left(z_{n}\right)\right)=0$, one shows that for each $n_{0}, \mu\left(F\left(z_{n}\right)\right) \leq \sum_{i \in z_{n_{0}}} b_{i}$. But note that

$$
\begin{aligned}
& F\left(\left(z_{n}\right)\right) \subseteq\left\{\left(m_{n}\right) \in K: \exists n m_{n} \in z_{n_{0}}\right\} \\
& \quad=\bigcup_{i \in z_{n_{0}}}\left\{\left(m_{n}\right) \in K: m_{n_{i}}=i \text { where } n_{i} \text { is the unique } n \text { with } i \in x_{n}\right\}
\end{aligned}
$$

and by the definition of $\mu$ this last set has measure not exceeding $\sum_{i \in z_{n_{0}}} b_{i}$. By its very definition $F$ is monotone. To see that its range is cofinal in $\mathcal{E}_{\mu}$ fix a closed measure zero subset $L$ of $K$. We can find now an increasing sequence $\left(k_{n}\right) \in \mathbb{N}^{\mathbb{N}}$ such that if we let $z_{n}=\left\{m \in x_{k_{n}}: \exists\left(m_{k}\right) \in L m_{k_{n}}=m\right\}$, then $\sum_{n} \sum_{i \in z_{n}} b_{i}<\infty$. Thus, $\left(z_{n}\right) \in D^{\prime}(I)$. It is easy to check that $L \subseteq F\left(\left(z_{n}\right)\right)$.

\section{Cofinal Subsets of ANALYTIC IDEALS}

A natural class of directed partial orders consists of analytic ideals of subsets of $\mathbb{N}$ with the inclusion order. We establish a result about definability of cofinal subsets of analytic ideals. It was proved by Zafrany in [15] that any analytic ideal $I$ contains a $G_{\delta}$ subset $G$ such that every element of $I$ is contained in the union of finitely many elements of $G$. Moreover, some analytic ideals have natural cofinal $G_{\delta}$ subsets. Consider, for example, a compact metric space $X$ with a countable basis $\left\{U_{n}: n \in \mathbb{N}\right\}$. Let $\mathcal{I}$ be an analytic $\sigma$-ideal of closed subsets of a compact metric space $X$. (So $\mathcal{I}$ is, in fact, $G_{\delta}$.) Then the ideal $I$ of subsets of $\mathbb{N}$ naturally associated with $\mathcal{I}$ by letting

$$
x \in I \text { iff } \exists K \in \mathcal{I} \forall n \in x U_{n} \cap K \neq \emptyset
$$

is analytic and has a natural $G_{\delta}$ cofinal family consisting of sets of the form $x_{K}=$ $\left\{n: U_{n} \cap K \neq \emptyset\right\}$ with $K \in \mathcal{I}$. We show that cofinal $G_{\delta}$ 's always exist. This result strengthens Zafrany's theorem mentioned above.

Theorem 8.1. Each analytic ideal of subsets of $\mathbb{N}$ has a cofinal $G_{\delta}$ subset.

Proof. According to [9, Theorem 2.1 and Theorem 3.4], we have three cases. (In the notation of [9], Case 2 below is $I_{1} \leq_{f} I$ and Case 3 is $\operatorname{Fin}^{\mathbb{N}} \leq_{f} I$.)

Case $1 . I$ is $F_{\sigma}$.

Then $I=\bigcup_{n} F_{n}$ with $F_{n}$ compact. Let

$$
F_{n}^{\prime}=\left\{\{0, \ldots, n\} \cup x: x \in F_{n}\right\} .
$$

Each $F_{n}^{\prime}$ is a compact subset of $I$ and for any sequence $x_{n}$ with $x_{n} \in F_{n}^{\prime}, x_{n} \rightarrow \mathbb{N}$. Thus, $\bigcup_{n} F_{n}^{\prime}$ is a $G_{\delta}$. Clearly it is cofinal in $I$.

Case 2. There exists a partition $\left\{d_{i}^{k}: i, k \in \mathbb{N}\right\}$ of $\mathbb{N}$ into finite non-empty sets such that for $y \subseteq \mathbb{N} \times \mathbb{N}$

$$
\bigcup\left\{d_{i}^{k}:(i, k) \in y\right\} \in I \text { iff } \exists k_{0} \forall(i, k) \in y k<k_{0} .
$$


Let $A_{n}=\left\{x \in I: \forall k \geq n \forall i d_{i}^{k} \nsubseteq \subseteq x\right\}$. Let $W=\left\{x \in 2^{\mathbb{N}}: \exists^{\infty} i x(i)=1\right\}$. Since $A_{n}$ is analytic, we can find a continuous surjection $g_{n}: W \rightarrow A_{n}$. Now define $g_{n}^{\prime}: W \rightarrow A_{n}$ by letting

$$
g_{n}^{\prime}(x)=\bigcup\left\{d_{i}^{k}: k<n\right\} \cup \bigcup\left\{d_{i}^{n}: x(i)=1\right\} \cup g_{n}(x) .
$$

We check that $g_{n}^{\prime}$ is a homeomorphic embedding. It is clearly continuous and since

$$
\left\{i: d_{i}^{n} \subseteq g_{n}^{\prime}(x)\right\}=\{i: x(i)=1\},
$$

it is injective. If $g_{n}^{\prime}\left(x_{k}\right) \rightarrow g_{n}^{\prime}(x)$ as $k \rightarrow \infty$, then, since the sets $d_{i}^{k}$ are finite,

$$
\left\{i: d_{i}^{n} \subseteq g_{n}^{\prime}\left(x_{k}\right)\right\} \rightarrow\left\{i: d_{i}^{n} \subseteq g_{n}^{\prime}(x)\right\} \text { as } k \rightarrow \infty .
$$

It follows that $x_{k} \rightarrow x$ and $\left(g_{n}^{\prime}\right)^{-1}$ is shown to be continuous. This implies that $A_{n}^{\prime}=g_{n}^{\prime}[W]$ are $G_{\delta}$. Moreover, if $x_{n} \in A_{n}^{\prime}$, then $x_{n} \rightarrow \mathbb{N}$, hence $B=\bigcup_{n} A_{n}^{\prime}$ is a $G_{\delta}$ as well. Since for each $y \in A_{n}$ we can find $y^{\prime} \in A_{n}^{\prime}$ with $y \subseteq y^{\prime}$ and since $B \subseteq I$, we see that $B$ is cofinal in $I$.

Case 3. There exists a partition $\left\{d_{i}^{k}: i, k \in \mathbb{N}\right\}$ of $\mathbb{N}$ into finite non-empty sets such that for $y \subseteq \mathbb{N} \times \mathbb{N}$

$$
\bigcup\left\{d_{i}^{k}:(i, k) \in y\right\} \in I \text { iff } \forall k \exists i_{0} \forall(i, k) \in y i<i_{0} .
$$

Let

$$
A=\left\{y \in I: \forall i, k d_{i}^{k} \not \subseteq y\right\} .
$$

The set $A$ is analytic, so we can find a continuous surjection $g: \mathbb{N}^{\mathbb{N}} \rightarrow A$. Consider the function $h: \mathbb{N}^{\mathbb{N}} \times \mathbb{N}^{\mathbb{N}} \rightarrow I$ whose value $h\left(z_{1}, z_{2}\right)$ is given by

$$
\bigcup\left\{d_{i}^{k}: i \leq z_{1}(k)\right\} \cup \bigcup\left\{d_{i}^{k}: z_{1}(k)+2 \leq i \leq z_{1}(k)+z_{2}(k)+2\right\} \cup g\left(z_{2}\right) .
$$

Let $B$ denote the range of $h$. Note that by the choice of the $d_{i}^{k}$ 's, $B$ is indeed included in $I$. In fact $B$ is cofinal in $I$. To see this, note that for any $y \in I, y=y_{1} \cup y_{2}$ such that $y_{2}$ does not contain any $d_{i}^{k}$ 's and, for some $z_{1} \in \mathbb{N}^{\mathbb{N}}$, if $d_{i}^{k} \subseteq y_{1}$, then $i \leq z_{1}(k)$. Pick now $z_{2}$ so that $y_{2}=g\left(z_{2}\right)$. Then $y=y_{1} \cup y_{2} \subseteq h\left(z_{1}, z_{2}\right)$.

Thus, it will suffice to show that $B$ is a $G_{\delta}$. To see this, it is enough to prove that $h$ is a homeomorphic embedding. Its continuity and injectivity follow immediately from the definition. To see continuity of the inverse of $h$, note that if $h\left(z_{1}^{n}, z_{2}^{n}\right) \rightarrow$ $h\left(z_{1}, z_{2}\right)$ as $n \rightarrow \infty$, then, by finiteness of the $d_{i}^{k}$ 's, $z_{1}^{n} \rightarrow z_{1}$ and $z_{2}^{n} \rightarrow z_{2}$.

\section{Questions}

The P-ideal $\mathcal{I}_{1 / n}$ is a Tukey maximal analytic P-ideal, in fact, more is true. Theorem 2B from [3] implies that for a basic order to be Tukey below $\mathcal{I}_{1 / n}$ it suffices that there exists on it a complete metric compatible with the topology and making the maximum operation uniformly continuous. Also NWD $\leq_{T} \mathcal{I}_{1 / n}$ is true [3]. Thus, the following questions seems justified.

Question 1. Is $\mathcal{I}_{1 / n}$ Tukey maximal among all analytic basic orders?

The next question is related to Proposition 6.8. 
Question 2. Is $\mathcal{Z}_{0}$ Tukey maximal among $\left((\text { Fin } \ltimes \text { Fin })^{+}, \emptyset \ltimes\right.$ Fin $)$-calibrated analytic P-ideals? Is it maximal among $\left((\text { Fin } \ltimes \text { Fin })^{+}, \emptyset \ltimes\right.$ Fin $)$-calibrated analytic basic orders?

The first part of the next question is an old problem of Fremlin. The second part asks for even more. It has to do with Proposition 6.3.

Question 3. Is NWD Tukey maximal among analytic $\sigma$-ideals of compact sets? Is NWD maximal among $\left(\right.$ Fin $\left.^{*}, \mathcal{P}(\mathbb{N})\right)$-calibrated analytic basic orders?

In [8, Theorem 6], a family of analytic P-ideals $I_{S}, S \subseteq \mathbb{N}$, is proved to fulfill $I_{S_{1}} \leq_{T} I_{S_{2}}$ iff $S_{1} \backslash S_{2}$ is finite. The next question asks whether this would persist after applying the functor $D$. An affirmative answer to this question would show that the Tukey order is very complicated among basic orders which are $\leq_{T}$ NWD and strictly Tukey above $\mathbb{N}^{\mathbb{N}}$. No two Tukey inequivalent elements are known there.

Question 4 . Is it true that $D\left(I_{S_{1}}\right) \leq_{T} D\left(I_{S_{2}}\right)$ iff $S_{1} \backslash S_{2}$ is finite?

The following question concerns the top part of the Tukey order among analytic P-ideals.

Question 5. Are there two Tukey inequivalent $F_{\sigma}$ P-ideals?

The next question is related to Proposition 4.3.

Question 6. Is it true that if $D$ is a locally compact, non-compact basic order, then $D \equiv_{T} \mathbb{N}$ or $\mathbb{N}^{\mathbb{N}} \leq_{T} D ?$

As the following slight modification of an example from [8, Example 1] demonstrates, if the above question has an affirmative answer, the proof will have to fully use the assumption that the maximum operation on a basic order is continuous. Define the partial order to be

$$
D=\left\{x \subseteq \mathbb{N} \times \mathbb{N}: \forall k \forall n\left|x \cap\left(\{k\} \times\left\{0, \ldots 2^{n}\right\}\right)\right| \leq n^{k}\right\}
$$

with inclusion as the order relation. By following the argument from [8], one checks that $D$ is not Tukey comparable with $\mathbb{N}^{\mathbb{N}}$ so it fails the property asked about in the question. It is, however, easy to see that $D$ is locally compact with the topology inherited from $2^{\mathbb{N} \times \mathbb{N}}$ and that it fulfils conditions (2) and (3) from the definition of basic orders. In fact, it satisfies Lemmas 3.1 and 3.2. Moreover, union is the least upper bound operation on $D$. It is Borel but it fails to be continuous.

The following question asks for strengthening of Theorem 7.7.

Question 7. Is $D\left(\mathcal{I}_{1 / n}\right)$ Tukey equivalent to $\mathcal{E}_{\mu}$ ? Is it Tukey equivalent to NWD?

\section{REFERENCES}

[1] J.P.R. Christensen, Topology and Borel Structure, North-Holland/Elsevier, 1974.

[2] I. Farah, Analytic Quotients, Mem. Amer. Math. Soc. 148(2000), no.702.

[3] D.H. Fremlin, The partially ordered sets of measure theory and Tukey's ordering, Note di Matematica 11(1991), 177-214.

[4] D.H. Fremlin, Families of compact sets and Tukey ordering, Atti. Sem. Mat. Fiz. 39(1991), $29-50$. 
[5] J.R. Isbell, Seven cofinal types, J. London Math. Soc. 4(1972), 651-654.

[6] A.S. Kechris, Classical Descriptive Set Theory, Springer, 1995.

[7] A.S. Kechris, A. Louveau, W.H. Woodin, The structure of $\sigma$-ideals of compact sets, Trans. Amer. Math. Soc. 301(1987), 263-288.

[8] A. Louveau, B. Veličković, Analytic ideals and cofinal types, Ann. Pure Appl. Logic 99(1999), 171-195.

[9] S. Solecki, Analytic ideals and their applications, Ann. Pure Appl. Logic 99(1999), 51-72.

[10] S. Todorcevic, Directed sets and cofinal types, Trans. Amer. Math. Soc. 290(1985), 711-723.

[11] S. Todorcevic, A classification of transitive relations on $\aleph_{1}$, Proc. London Math. Soc. 73(1996), 501-533.

[12] S. Todorcevic, Analytic gaps, Fund. Math. 150(1996), 55-66.

[13] S. Todorcevic, Definable ideals and gaps in their quotients, in Set Theory (Curacao 1995, Barcelona 1990), pp. 213-226, Kluwer, Dordrecht 1998.

[14] J.W. Tukey, Convergence and uniformity in topology, Ann. Math. Studies, 1(1940), Princeton U.P.

[15] S. Zafrany, On analytic filters and prefilters, J. Symb. Logic 55(1990), 315-322.

Department of Mathematics, University of Illinois, 1409 W. Green St., Urbana, IL 61801, USA

E-mail address: ssolecki@math.uiuc.edu

Universite Paris 7-C.N.R.S., UMR 7056, 2 Place Jussieu, 75251 Paris Cedex 05, France

E-mail address: stevo@math.jussieu.fr 